\title{
„den fliegenden măren zeglauben kein ursach sîe" - Das Mandat Maximilians I. vom 22. April 1499 in seiner propagandistischen Dimension
}

\author{
Tobias Riedmann \\ Kerngebiet: Österreichische Geschichte \\ eingereicht bei: ao. Univ.-Prof. Dr. Heinz Noflatscher \\ eingereicht im: SoSe 2018 \\ Rubrik: BA-Arbeit
}

\begin{abstract}
"den fliegenden mâren zeglauben kein ursach sîe" - The mandate of Maximilian I of April $22^{\text {nd }} 1499$ in its propagandistic dimensions

This paper deals with the propaganda used by Emperor Maximilian I during the Swiss/Swabian War of 1499, and in particular the mandate of April $22^{\text {nd }}$. In accordance with the genuine communication situation of the Early Modern period, it was necessary to adapt the respective framework conditions to the corresponding time segment through a theoretical determination of the communication situation. Based on these theoretical premises, this study analyses original sources according to their propagandistic dimensions and investigates the context, the addressed audience and the declared purpose correspondingly.
\end{abstract}

\section{Einleitung}

"Alle Menschen kommen ja dadurch zu einer Überzeugung, daß sie, wenn sie entscheiden, sich selbst in eine bestimmte Stimmung versetzt sehen oder daß sie glauben, die Redner seien mit diesem oder jenem Charakterzug ausgestattet, oder daß sie vor einem Beweis stehen." 
Wie Aristoteles in seinem dritten Buch zur Rhetorik darlegt, ist es die Rede, welche die Öffentlichkeit zu einer bestimmten Verhaltens- oder Denkweise bewegen soll.2 Diese Form der Kommunikation, die selbstverständlich gewissen Voraussetzungen unterliegt, kann als zeitlose Begleiterscheinung von kriegerischen Konflikten gesehen werden, womit sie auch auf den Schwabenkrieg von 1499 zutreffen würde, der sich durch zahlreiche Propagandaaktionen beider Seiten auszeichnet. ${ }^{3}$ Eine wesentliche Neuerung, die sich hier gegenüber den vorherigen Jahrhunderten konstatieren lässt, ist indessen die Verwendung von Druckmedien zur umfangreichen Verbreitung der eigenen Meinung in der Öffentlichkeit. Gleichzeitig beeinflusste diese sogenannte Medienrevolution der Frühen Neuzeit nachhaltig die Art der Kommunikation. ${ }^{4}$

Insbesondere Maximilian I. scheint die Vorteile dieser neuen Medien für seine Politik erkannt zu haben. ${ }^{5}$ Gleichwohl konstatiert Werner Meyer, dass „[...] hinter der drohenden Rhetorik Maximilians und seiner Leute keine konkreten Absichten und schon gar keine ausreichenden Mittel [steckten]."6 Ein prominentes Beispiel für die Propaganda Maximilians ist das Mandat vom 22. April 1499. Scheinbar trifft diese Beobachtung auch zu, wenn die Eidgenossen als hauptsächlicher Adressatenkreis dieser Drucke betrachtet werden. Sobald das Mandat aber gemäß seinem Inhalt, seiner Verbreitung unter den süddeutschen Ständen und der Intention Maximilians, seine Macht in den Vorlanden auszubauen, analysiert wird, ergeben sich unterschiedliche Fragen: Handelte es sich dabei per Definition überhaupt um Propaganda? Versuchte Maximilian mit seinen publizierten Mandaten, allen voran dem erwähnten Mandat, die Handlungen und Meinungen der süddeutschen Stände bezüglich ihres Verhaltens im Konflikt zu manipulieren? Gelang inm dies mit seiner Propaganda? Welche Rolle spielten dabei die jeweiligen politischen Hintergründe für die Akteur*innen der Kommunikation? Ausgehend von diesen Fragen kann auf Grundlage von Quellenstudien und in Abgleich mit der Forschungsliteratur folgende Hypothese aufgestellt werden: Die offizielle habsburgische Propaganda im Schwabenkrieg zielte weniger auf eine reine Diffamierung der Eidgenossen ab, sondern versuchte entsprechend der Funktion von Propaganda die adressierte Öffentlichkeit zu einem gewissen Verhalten im Konflikt zu bewegen, wobei der gemeinsame Sinnhorizont eine wesentliche Rolle spielte.?

2 Zur Verortung von Aristoteles im Propagandadiskurs: Klaus Merten, Struktur und Funktion von Propaganda, in: Publizistik 45 (2000), Heft 2, S. 143-162, hier S. 144.

3 Volker Reinhardt, Die Geschichte der Schweiz. Von den Anfängen bis heute, München 2014, S. 140; Gutmann weist in seiner Einleitung auf die unterschiedliche Bezeichnung «Schwaben- oder Schweizerkrieg» hin. In dieser Arbeit wird der Lesbarkeit halber einzig die Bezeichnung Schwabenkrieg verwendet: Andre Gutmann, Die Schwabenkriegschronik des Kaspar Frey und ihre Stellung in der eidgenössischen Historiographie des 16. Jahrhunderts (Veröffentlichungen der Kommission für Geschichtliche Landeskunde in Baden-Württemberg Reihe B, Forschungen 176,1), Stuttgart 2010, S. 1.

4 Überblicksmäßig zum Begriff der Medienrevolution: Andreas Würgler, Medien in der frühen Neuzeit (Enzyklopädie deutscher Geschichte 85), München 2009, S. 68-69.

$5 \quad$ Heinz-Dieter Heimann, Die Habsburger. Dynastie und Kaiserreiche, München 20015 , S. 47.

6 Werner Meyer, Krisen, Korruption und Kampfbegierde. Der politische, ideologische und emotionale Konfliktrahmen des Schwabenkrieges von 1499, in: Historischer Verein des Kantons Solothurn (Hrsg.), Gedenkschrift 500 Jahre Schlacht bei Dornach 1499-1999 (Jahrbuch für solothurnische Geschichte 72), Solothurn 1999, S. 10-52, hier S. 51.

7 Selbstverständlich kann im Rahmen dieser Bachelorarbeit nicht auf die Gesamtheit, der von der habsburgischen Partei ausgegangenen Propaganda eingegangen werden, weshalb gerade diffamierende Lieder, Flugschriften, etc., die keinen direkten Verweis auf Maximilian haben, nicht zur offiziellen Propaganda gezählt werden. 
Um die aufgestellte Hypothese angemessen bearbeiten zu können, bedarf es zunächst einer methodischen Festlegung, die verhindern soll, dass diese Arbeit durch anekdotische Beobachtungen einen vorwissenschaftlichen Charakter erhält. ${ }^{8}$ Die Basis hierfür umfassen unterschiedliche Ansätze zur Kommunikations- und Propagandatheorie. Für diesen ersten Bereich sind insbesondere Autor*innen wie Klaus Merten, Thymian Bussemer, Birgit Studt und Eva-Maria Schnurr hervorzuheben. Die ersten beiden Wissenschaftler liefern dabei die theoretische Grundlage im Allgemeinen, wohingegen Studt und Schnurr vor kurzem mit ihren Arbeiten derartige Theorien an den historischen Bereich angewandt haben. Auf diesen theoretischen Prämissen aufbauend versucht die Arbeit, durch die Analyse einer historischen Quelle - das Mandat König Maximilians I. vom 22. April 1499 - die hergestellte Kommunikationssituation zu erschließen und die Quelle auf ihre Intention und Wirkung zu hinterfragen. Damit soll gezeigt werden, dass die offizielle habsburgische Propaganda Maximilians I. im Schwabenkrieg viel eher als Instrument zur Festigung der landesherrlichen Strukturen verstanden werden muss als zur grobschlächtigen Verunglimpfung der Eidgenossen. ${ }^{9}$

Um dieses Postulat untermauern zu können, stützt sich diese Bachelorarbeit in ihren Überlegungen auf wesentliche Forschungsarbeiten zu Vorderösterreich und zur Propaganda Maximilians. Gemeint sind damit vor allem die Ausführungen von Alois Niederstätter, Werner Meyer, Volker Press, Horst Carl und Jan-Dirk Müller. Gerade Letzterer hat sich in den letzten Jahren als Kenner der politischen Propaganda von Maximilians I. hervorgetan. Hingegen widmen sich Niederstätter und Meyer den politischen und ideologischen Rahmenbedingungen des Schwabenkrieges. Schließlich sind Press und Carl vor allem im Zusammenhang mit der habsburgischen Präsenz im süddeutschen Raum zu nennen. In einem an die Eruierung der Quelle und des Kontextes anschließenden Kapitel werden die wesentlichen Argumente zusammengefügt und diskutiert.

\section{Methodische Überlegungen}

Obwohl die interdisziplinäre Beschäftigung mit dem Themenkomplex der Kommunikation bereits eine lange Tradition aufweist, hat die Geschichtswissenschaft diesen Bereich relativ spät für sich entdeckt. ${ }^{10}$ Insbesondere für die Erforschung der Frühen Neuzeit hängt diese erfolgte Annäherung an den Forschungsgegenstand mit der Behandlung der Epochenschwelle um 1500 als kommunikationsverändernde Größe zu-

Thymian Bussemer, Propaganda. Konzepte und Theorien, Wiesbaden 2005, S. 22.

Zum Prozess der «Formierung der Lande» von Sigmund bis Maximilian: Dieter Speck, Kleine Geschichte Vorderösterreichs, Karlsruhe 2016², S. 91-103.

10 Zur Forschungsgeschichte innerhalb der Geschichtswissenschaften: Wolfgang Behringer, Kommunikation, in: EdN, Bd. 6, Stuttgart 2007, Sp. 995-1018; Klaus Beck, Kommunikationsforschung, in: Günter Bentele/HansBernd Brosius/Otfried Jarren (Hrsg.), Lexikon Kommunikations- und Medienwissenschaft, Wiesbaden 20132, S. 158-159. 
sammen. ${ }^{11}$ Fast allen Ansätzen ist der Grundsatz gemein, dass spätestens ab Mitte des 15. Jahrhunderts ein kommunikationsgeschichtlicher Wandel einsetzt, der Auswirkungen auf diverse gesellschaftliche Teilbereiche hat.12 Tatsächlich handeln die meisten Diskussionen vor allem vom Verhältnis der Geschichtswissenschaften zur Medien- und Kommunikationstheorie. ${ }^{13}$ Dazu gehören auch die Teilbereiche der politischen Kommunikation und Propaganda. Da die später vorgestellten Quellen tendenziell dieser frühneuzeitlichen Entwicklung zugeordnet werden können, muss vorab geklärt werden, unter welchen theoretischen Voraussetzungen diese zu betrachten sind. Insofern ist aufgrund des direkten zeitlichen Umfeldes zur Medien-/Kommunikationsrevolution von einem bestimmten Wesen von Öffentlichkeit auszugehen. ${ }^{14}$

\subsection{Kommunikation und Öffentlichkeit}

Die Erforschung des im 15. und 16. Jahrhundert einsetzenden Wandels bedient sich zweierlei Begrifflichkeiten: zum einem der Kommunikation und zum anderen der Öffentlichkeit. Gerade weil die Begriffsbestimmung dieser Termini aufgrund unterschiedlicher Kommunikationsmodelle-/theorien heterogen ausfällt, sind sie innerhalb des Untersuchungszeitraumes mit Sorgfalt zu verwenden. ${ }^{15}$

Zunächst ist es erforderlich für den Kommunikationsbegriff einige Aussagen zu treffen: Der Tenor der geistes- und sozialwissenschaftlichen Forschung ist, dass unter Kommunikation ein soziales Phänomen verstanden wird, dem ein Prozess der Informationsübertragung zugrunde liegt, der mindestens zwischen zwei Menschen stattinden muss. ${ }^{16}$ Außerdem lassen sich laut Beck in den meisten theoretischen Modellen fünf Gemeinsamkeiten feststellen: „(1) Sender, (2) Botschaft,

11 Zum Forschungsschwerpunkt bzgl. des Druckes als "agent of change“: Elizabeth L. Eisenstein, The printing revolution in early modern Europe, Cambridge 2005²; Für die deutschsprachige Forschung: Michael Giesecke, Der Buchdruck in der frühen Neuzeit. Eine historische Fallstudie über die Durchsetzung neuer Informations- und Kommunikationstechnologien, Frankfurt am Main 20064; Laut Werner Faulstich ist dieser Prozess aber nicht auf den Buchdruck allein zu reduzieren:Werner Faulstich, Medien zwischen Herrschaft und Revolte. Die Medienkultur der frühen Neuzeit (1400-1700) (Die Geschichte der Medien 3), Göttingen 1998.

12 Wolfgang Behringer postuliert die These, dass erst mit dem Aufkommen des Postwesens die Kommunikationsrevolution eingeleitet wurde: Wolfgang Behringer, Im Zeichen des Merkur. Reichspost und Kommunikationsrevolution in der Frühen Neuzeit (Veröffentlichungen des Max-Planck-Instituts für Geschichte 189), Göttingen 2003; Vereint werden einige dieser Beiträge im Sammelband von Johannes Burkhardt und Christine Werkstetter. In der dazugehörigen Podiumsdiskussion spricht sich Winfried Schulze letztlich trotz der unterschiedlichen Standpunkte des Medienwandels für eine pragmatische „Kombi-Lösung“ aus, wie sie Wolfgang Reinhard aufgreift: Johannes Burkhardt/Christine Werkstetter, Begann die Neuzeit mit dem Buchdruck? Ist die Ära der Typographie im Zeitalter der digitalen Medien endgültig vorbei? Podiumsdiskussion unter der Leitung von Winfried Schulze, in: Johannes Burkhardt/Christine Werkstetter (Hrsg.), Kommunikation und Medien in der Frühen Neuzeit (HZ. Beihefte n.F. 41), München 2005, S. 11-39; Wolfgang Reinhard, Rezension zu: Johannes Burkhardt/ Christine Werkstetter (Hrsg.), Kommunikation und Medien in der Frühen Neuzeit (HZ. Beihefte. NF, 41), München 2005, in: Zeitschrift für Historische Forschung 34 (2007), Heft 4, S. 684-686, hier S. 685.

13 Fabio Crivellari/Kay Kirchmann u. a., Einleitung: Die Medialität der Geschichte und die Historizität der Medien, in: Fabio Crivellari/Sven Grampp u. a. (Hrsg.), Die Medien der Geschichte. Historizität und Medialität in interdisziplinärer Perspektive (Historische Kulturwissenschaft 4), Konstanz 2004, S. 9-48, hier S. 14.

14 Jürgen Habermas, Strukturwandel der Öffentlichkeit. Untersuchungen zu einer Kategorie der bürgerlichen Gesellschaft, Frankfurt am Main 2015'14; Friedrich Jaeger, Kultur, in: EdN, Bd. 7, Stuttgart 2008, Sp. 253-281, hier Sp. 268.

15 Klaus Beck, Kommunikation, in: Bentele/Brosius/Jarren (Hrsg.), Lexikon Kommunikations- und Medienwissenschaft, Wiesbaden 20132, S. 155-156, hier S. 155. 
(3) Empfänger, (4) Übermittlungseinrichtung [Medium], (5) Wirkung."17 Nichtsdestotrotz bedarf es einer gewissen begrifflichen Spezifizierung, um mit den gewählten Konzepten arbeiten zu können.

Eine erste Annäherung an den Begriff bietet das systemtheoretische Modell von Niklas Luhmann, das von hoher Bedeutung für die Erforschung der Kommunikation der Frühen Neuzeit ist. So hat diese Herangehensweise jüngst bei Wissenschaftler*innen wie Rudolf Schlögl und Barbara Stollberg-Rilinger ihren Anklang gefunden. ${ }^{18}$ Schnurr hat die Quintessenz des luhmann'schen Kommunikationsbegriffes deutlich auf den Punkt gebracht:

„Kommunikation ist [die] gemeinsame Aktualisierung von Sinn: Die Botschaft des Senders erhält ein Informationsangebot, das der Empfänger auf Grundlage seines Vorwissens, [...] interpretiert und für sich als Information erkennt und versteht, so dass eine weitere Kommunikation daran anschließen kann."19

Vereinfacht dargestellt, gilt die Kommunikation als "geglückt", wenn darauf reagiert wird. Hiermit wird vermieden, Wirkungen in eine lineare Übertragung hineinzuinterpretieren. ${ }^{20}$ Aus historischer Sicht ergibt sich daraus jedoch ein quellenbezogenes Problem. Für viele vormoderne Kommunikationssituationen sind schlicht und einfach zu wenig Quellen überliefert, mit denen sich das Modell vollends anwenden ließe.

Ein weiterer nicht unwesentlicher Kritikpunkt zur Anwendbarkeit dieses Modells für den gewählten Untersuchungszeitraum ist folgender: Luhmann differenziert in seiner soziologischen Herangehensweise die Gesellschaft in verschiedene soziale Systeme (Recht, Wirtschaft, etc.), die sich durch Kommunikationsformen unterscheiden können und in der Moderne vorwiegend funktional agieren. ${ }^{21}$ Da aber eine Ausdifferenzierung der funktionalen Systeme sich erst ab dem 18. Jahrhundert historisch nachweisen lässt, leidet auch hier die Angemessenheit des Modells. ${ }^{22}$ Aufgrund dieses theoretischen Problems haben sich bereits Forscher*innen, wie Schnurr, für eine durch andere Theorien angereicherte modulare Begrifflichkeit entschieden, woran sich diese Bachelorarbeit orientiert. ${ }^{23}$ Auch die von Beck postulierten Meta-Gemeinsamkeiten der Kom-

17 Beck, Kommunikation; Bereits 1948 beschrieb Harold Lasswell in einer formelhaften Weise den Begriff der Kommunikation: „[A] convenient way to describe an act of communication is to answer the following questions: Who, Says What, In Which Channel, To Whom, With What Effect?" aus: Harold D. Lasswell, The structure and function of communication in society, in: Lyman Bryson (Hrsg.), The Communications of Ideas, New York 1948, S. 117-130, hier S. 117.

18 Rudolf Schlögl, Anwesende und Abwesende. Grundriss für eine Gesellschaftsgeschichte der Frühen Neuzeit, Konstanz 2014; Für eine Einarbeitung der Theorie Luhmanns: Barbara Stollberg-Rilinger, Des Kaisers alte Kleider. Verfassungsgeschichte und Symbolsprache des Alten Reiches, München $2013^{2}$.

19 Eva-Maria Schnurr, Religionskonflikt und Öffentlichkeit. Eine Mediengeschichte des Kölner Kriegs (1582 bis 1590) (Rheinisches Archiv 154), Köln 2009, S. 41; Niklas Luhmann, Soziale Systeme. Grundriss einer allgemeinen Theorie, Frankfurt am Main 1987², S. 194 und S. 203.

20 Damit ist das Lasswell'sche Kommunikationsmodell gemeint: Lasswell, Communication in society, S. 117

21 Zum Begriff des Systems: Luhmann, Soziale Systeme, S. 33; Zur Klärung der Begriffe das Glossar von Claudio Baraldi/Giancarlo Corsi/Elena Esposito (Hrsg.), GLU. Glossar zu Niklas Luhmanns Theorie sozialer Systeme, Frankfurt am Main 2015.

22 Zur Grenzziehung: Christoph Reinfandt, Systemtheorie, in: Ansgar Nünning (Hrsg.), Metzler Lexikon Literatur- und Kulturtheorie. Ansätze - Personen - Grundbegriffe, Stuttgart-Weimar 2013, S. 735-737, hier S. 735. 
munikationsmodelle sind in der systemtheoretischen Definition wiederzufinden und erscheinen deshalb für die Analyse nützlich. ${ }^{24}$ Weitere Implikationen für die Untersuchung ergeben sich aus dem später zu behandelnden begrifflichen Instrumentarium der Propagandaforschung. ${ }^{25}$

Deutliche Anleihen an der Systemtheorie Luhmanns sind ebenfalls bei Schlögl zu erkennen. ${ }^{26}$ Dieser versucht, in seiner Forschung die kommunikativen Figurationen der Frühen Neuzeit zu erklären, die infolge der Kommunikations-/Medienrevolution des 15. und 16. Jahrhunderts verändert wurden. ${ }^{27}$ Darunter ist zu verstehen, dass vormals ortsabwesende Akteur*innen, die nicht direkt der Kommunikation beiwohnten, zusehends in die Sinn-Aktualisierung mit einbezogen werden konnten. Denn Schlögl führt die Stärkung der Organisationsfähigkeit sozialer Konfigurationen auf den „intensiveren Gebrauch von Schrift [...] als Kommunikationsmedium zurück."28 Gemeint ist hier vor allem der Faktor der entgrenzten Kommunikation, die sich vom vormaligen face-to-face Prinzip zu lösen beginnt. ${ }^{29}$ Dieser Umstand gewinnt an Brisanz, wenn die steigende Verwendung von technisch neuen Medien in der politischen Kommunikation bedacht wird. Denn folglich war es vermehrt möglich, soziale Strukturen durch schriftgestützte Medien ortsunabhängig durchzusetzen. ${ }^{30}$

Eine weitere definitorische Erläuterung bedarf der Begriff der Öffentlichkeit. Dieser ist von eminenter Bedeutung für die Frühe Neuzeit und muss gemäß Wolfgang Schmale, Clemens Zimmermann und Gunter Mahlerwein in seinem Wesen als dynamisch und eng mit der Gesellschaft verknüpft betrachtet werden. ${ }^{31}$ Ihnen zufolge bildete sich die gesellschaftliche Segmentierung auch in der Öffentlichkeit ab, wobei die Trennung nicht strikt vollzogen werden darf. ${ }^{32}$ Schnurr spricht in diesem Zusammenhang von sogenannten Teilöffentlichkeiten, schränkt aber ein, dass Personen nie nur einer davon angehörten. ${ }^{33}$ Genau diese Annahme wird jedoch von Schlögl hinterfragt, da er meint, dass durch eine unscharfeVerwendung desÖffentlichkeitsbegriffes, der Untersuchungsgegenstand zusehends verschwimmt. ${ }^{34}$

\footnotetext{
24 Schnurr, Religionskonflikt, S. 42.

25 Gerhard Vowe, Politische Kommunikation, in: Bentele/Brosius/Jarren (Hrsg.), Lexikon Kommunikations- und Medienwissenschaft, Wiesbaden 201322, S. 266-268, hier S. 266.

26 Schlögl, Anwesende, S. 29-35; Rudolf Schlögl, Kommunikation und Vergesellschaftung unter Anwesenden. Formen des Sozialen und ihre Transformation in der Frühen Neuzeit, in: Geschichte und Gesellschaft 34 (2008), Heft 2, S. 155-224; Rudolf Schlögl, Politik beobachten. Öffentlichkeit und Medien in der Frühen Neuzeit, in: Zeitschrift für Historische Forschung 35 (2008), Heft 4, S. 581-616.

27 Schlögl, Anwesende, 2014.

28 Schlögl, Kommunikation, S. 202.

29 Ute Frevert, Politische Kommunikation und ihre Medien, in: Ute Frevert/Wolfgang Braungart (Hrsg.), Sprachen des Politischen. Medien und Medialität in der Geschichte, Göttingen 2004, S. 7-19, hier S. 16.

30 Rudolf Schlögl, Medien der Macht und des Entscheidens. Schrift und Druck im politischen Raum der europäischen Vormoderne (14.-17. Jahrhundert), Eine Einleitung, in: Jan Marco Sawilla/Rudolf Schlögl (Hrsg.), Medien der Macht und des Entscheidens. Schrift und Druck im politischen Raum der europäischen Vormoderne (14.-17. Jahrhundert) (The formation of Europe 5), Hannover 2014, S. 7-32, hier S. 9-11.

31 Wolfgang Schmale/Clemens Zimmermann/Gunter Mahlerwein, Öffentlichkeit, in: EdN, Bd. 9, Stuttgart 2009, Sp. 358-367, hier Sp. 358.

32 Ebd

33 Schnurr, Religionskonflikt, S. 45-46.

34 Schlögl, Politik, S. 583.
} 
Ein weiteres Problem ergibt sich aus der Projektion des Begriffes in die Vergangenheit. Schnurr weist deshalb in ihrer Einleitung daraufhin, dass das Konzept begriffsgeschichtlich erst nach 1750 greifbar ist. Ihr zufolge ist deswegen mit dem Adjektiv öffentlich zu operieren, welches auf die Wahrnehmbarkeit, Zugänglichkeit und Bekanntheit verweist. ${ }^{35}$ Gerade im Zusammenhang mit der politischen Kommunikation und der Propaganda ist nach diesen drei Charakteristika zu fragen. So argumentieren Jonathan Auerbach und Russ Castronovo, dass Propaganda nur dann wirklich Propaganda ist, wenn sie auch wahrgenommen werden kann. Mithilfe der Drucktechnik entstand während der Medienrevolution eine neue Form der Öffentlichkeit, die neben der faceto-face Kommunikation existierte. ${ }^{36}$ Aus Überlieferungsgründen können bestimmte mobile Quellen, wie Flugblätter, Lieder etc. jedoch nur schwierig einer Öffentlichkeit zugeordnet werden, da sich diese unabhängig der Literalität verbreiteten und damit Öffentlichkeit erschufen. Die Zirkulation der Propagandamedien determiniert folglich deren Tragweite und ermöglicht ein räumliches Abstecken der Öffentlichkeit. ${ }^{37}$ Abhängig davon muss bedacht werden, dass Propaganda gezielt versucht eine bestimmte Öffentlichkeit herzustellen. ${ }^{38}$

\subsection{Propaganda in der Frühen Neuzeit?}

In kurzer Form ist es gleichfalls nötig, die Begrifflichkeit der Propaganda zu diskutieren. ${ }^{39}$ Um die dazugehörigen Facetten zu erfassen, entwirft der Kommunikationswissenschaftler Bussemer eine "Super-Definition" des Propagandabegriffs, in der er Propaganda als eine „in der Regel medienvermittelte Formierung handlungsrelevanter Meinungen und Einstellungen politischer oder sozialer Großgruppen durch symbolische Kommunikation und als Herstellung von Öffentlichkeit zugunsten bestimmter Öffentlichkeiten" definiert. ${ }^{40}$ Doch auch in diesem Fall ist es schwierig, die vorgeschlagene Definition eindeutig auf die Frühe Neuzeit umzulegen. ${ }^{41}$ Hierfür bedarf es einiger Präzisierungen, wie sie Bussemer deutlich macht. ${ }^{42}$ Diese werden aus unterschiedlichen Gründen benötigt: Sei es aus begriffsgeschichtlichen Gründen, allgemeinen Definitionsproblemen, einer veränderten Medienlandschaft oder dem Umstand der differenzierten Kommunikationskultur heraus. ${ }^{43}$

35 Schnurr, Religionskonflikt, S. 33-36.

36 Schlögl, Politik, S. 585.

37 Auerbach und Castronovo postulieren insgesamt 13 Thesen über das Wesen der Propaganda: Jonathan Auerbach/Russ Castronovo, Introduction: Thirteen Propositions about Propaganda, in: Jonathan Auerbach/Russ Castronovo (Hrsg.), The Oxford handbook of propaganda studies, New York 2013, S. 1-18, hier S. 5. Birgit Studt, Geplante Öffentlichkeit. Propaganda, in: Konstanzer Arbeitskreis für mittelalterliche Geschichte (Hrsg.), Politische Öffentlichkeit im Spätmittelalter (Vorträge und Forschungen 75), Konstanz 2011, S. 203-236, hier S. 234.

39 Bussemer, Propaganda, S. 22.

40 Ebd., S. 29-30.

41 Schnurr, Religionskonflikt, S. 371-374.

42 Bussemer, Propaganda, S. 30.

43 Zu den allgemeinen Definitionsproblemen: Sabine Doering-Manteuffel, Informationsstrategien: Propaganda, Geheimhaltung, Nachrichtennetze. Einleitung, in: Burckhardt/Werkstetter (Hrsg.) Kommunikation und Medien in der Frühen Neuzeit (HZ. Beihefte n.F. 41), München 2005, S. 367-384, hier S. 368; Bussemer, Propaganda, S. 24-29; So meinen Burkhardt und Werkstetter in ihrer Einleitung, dass sich diese Kultur der Kommunikation am Beginn der Neuzeit multimedial zu entwickeln begann: Johannes Burkhardt/Christine Werkstetter, Die Frühe Neuzeit als Medienzeitalter und ihr kommunikatives Spektrum. Einleitung, in: Burckhardt/Werkstetter (Hrsg.) Kommunikation und Medien in der Frühen Neuzeit (HZ. Beihefte n.F. 41), München 2005, S. 1-7, hier S. 2. 
Wenn zunächst ein Blick in die Begriffsgeschichte geworfen wird, fällt auf, dass sich das Substantiv Propaganda als terminus technicus erst ab dem Anfang des 17. Jahrhunderts, genauer im Zusammenhang mit der Sacra congregatio de propaganda fide (1622 gegründet), historisch mit dem Sinn der Verbreitung von Ideen nachweisen lässt. ${ }^{44}$ Vom damaligen Zeitpunkt bis zum Ende des 18. Jahrhunderts wurde das Wort hauptsächlich für die christliche Missionstätigkeit verwendet, weshalb es in seiner modernen Prägung kaum direkt auf die frühneuzeitliche politische Kommunikation umgelegt werden sollte. Wolfgang Schieder und Christof Dipper lokalisieren schließlich ab 1780 einen begriffsgeschichtlichen Wandel, infolgedessen der Propagandabegriff eine neue Bedeutung erhält, die ähnlich dem der Modernen ist und im Zuge der Französischen Revolution vollständig zum Tragen kommt. ${ }^{45}$ Obwohl sich der Begriff nicht vor 1622 festmachen lässt, bedeutet das nicht, dass es keine Kommunikationsformen vor diesem Zeitpunkt gab, die in ihrer Struktur und Funktion mit dem Propaganda Begriff gleichzusetzen sind. Studt Birgit hat auf diesen Umstand kürzlich hingewiesen. ${ }^{46}$ Auch der Kommunikationswissenschaftler Klaus Merten verweist darauf, dass bereits in der aristotelischen Rhetorik diese Grundform der persuasiven Kommunikation enthalten ist. Generell korreliert Propaganda stark mit politischer Macht - ein Umstand, der sich historisch betrachtet kaum ändert. ${ }^{47}$

Den Versuch einer ersten Systematisierung unternahm 1934 Harold D. Lasswell, der gleichzeitig als einer der Väter der Kommunikationswissenschaften gewertet wird. ${ }^{48}$ Lasswell verstand Propaganda zunächst noch als Überbegriff, unter dem er unterschiedliche persuasive Phänomene subsumierte. Die Funktion der Propaganda erkannte er dabei in der Beeinflussung der menschlichen Handlungen durch die Manipulation der Darstellungen in der Öffentlichkeit. ${ }^{49}$ Inzwischen hat dieses Grundverständnis zahlreiche Weiterentwicklungen erfahren, die größtenteils Propaganda als Kommunikationssituation aufgreifen und nicht bloß als reine Textsorte. Neuerdings werden die Kriterien für Propaganda differenzierter ausgelegt, um sie von anderen kommunikativen Techniken abzugrenzen. Merten vertritt die Ansicht, dass Propaganda im Kontrast zur Werbung und den public relations in ihrer Intention keine positiven Assoziationen hervorruft, sondern einen totalitären Anspruch vertritt, der eine Sanktionierung bei Nichtbefolgung impliziert.50 Vor dem Hintergrund des gewählten Untersuchungsfeldes gewinnt dieser Aspekt eine besondere Konnotation, den es zu berücksichtigen gilt.

Weiters unterscheidet Merten zwischen religiöser, politischer und Produkt-Propaganda, wobei für diese Arbeit insbesondere die politische Propaganda von Relevanz ist. ${ }^{51}$

44 Wolfgang Schieder/Christof Dipper, Propaganda, in: Otto Brunner/Werner Conze/Reinhart Koselleck (Hrsg.), Geschichtliche Grundbegriffe. Historisches Lexikon zur politisch-sozialen Sprache in Deutschland, Bd. 5, Stuttgart 1984, S. 69-88, hier S. 69; Bussemer, Propaganda, S. 25; Studt, Öffentlichkeit, S. 207.

45 Schieder/Dipper, Propaganda, S. 71; Studt, Öffentlichkeit, S. 207.

46 Studt, Öffentlichkeit, S. 207.

47 Merten, Propaganda, S. 144-145; Zur Propagandaproduktion durch die Obrigkeit: Schnurr, Religionskonflikt, S. 371.

48 Merten, Propaganda, S. 146.

49 Ebd.; Studt, Öffentlichkeit, S. 208

50 Merten, Propaganda, S. 150.

51 Ebd., S. 153-155. 
Hierfür formuliert er fünf Strukturmerkmale, die für die Analyse dieser Arbeit von Belang sind: (1) Das Alleinstellungsmerkmal eines/r mächtigen Individuums/Gruppe, (2) die Verhaltensprämisse des Gehorsams, (3) der Ausschließungscharakter Dritter, (4) die Voraussagung von positiven oder negativen Folgen, (5) die Nichtprüfbarkeit dieser Folgen. ${ }^{52}$ Zwei nicht unwesentliche Details hierzu stellen die Abgrenzung zur modernen Propagandastruktur und die Anwendung von Macht in Relation zur Propaganda dar. Beide Faktoren sind durch die bestehende Öffentlichkeit bzw. den Kommunikationsraum zu erklären. ${ }^{53}$ So hat Studt in ähnlicher Weise die Grundlage von Merten auf einen historischen Untersuchungszeitraum angewandt. Im Wesentlichen übernimmt sie die von Merten präsentierten Feststellungen zur Propaganda, beschränkt sich in ihrer Rezeption aber auf die ersten drei Punkte von Merten. ${ }^{54}$ Die Rückstellung der vierten und fünften Feststellung, die sich einerseits auf die ethische Bedenklichkeit und andererseits auf die Unterscheidungskriterien gegenüber der Werbung und public relations beziehen, werden von ihr aufgrund der differenzierten Situation in der Frühen Neuzeit außer Acht gelassen. Studt meint ebenfalls, dass die Androhung von Gewalt bzw. Sanktionen in der vormodernen Gesellschaft bedingt durch ein fehlendes staatliches Gewaltmonopol anderen Voraussetzungen unterlag..$^{55}$

\subsection{Medien der Propaganda}

Ein letzter Punkt, der in diesem Kapitel noch Erwähnung findet, ist eine kurz gefasste Bestimmung der Medien der Propaganda, denn innerhalb des Kommunikationsprozesses nimmt das Medium als Übermittlungseinrichtung eine hervorgehobene Stellung ein. Nicht ohne Grund betitelte einst Marshall McLuhan bewusst ironisch sein Buch mit "The medium is the massage [sic!]“56 Denn Medien sind viel mehr als ihre rein technischen Träger. Als Träger von Information zwischen Sender und Empfänger kann der Medienbegriff auf die unterschiedlichsten gesellschaftlichen Bereiche angewandt werden. ${ }^{57}$ Oft taucht in diesem Zusammenhang der Begriff des Massenmediums auf, der jedoch für die Frühe Neuzeit kaum anwendbar ist, da Medien zumeist im Rahmen bestimmter Öffentlichkeit zirkulieren, welche selten als "Masse" zu bezeichnen sind. ${ }^{58}$

Bewusst wird hier aber auf eine weitere begriffliche Ausführung verzichtet, um sich den für diese Arbeit relevanten Medien anzunehmen. Damit gemeint sind die ab Mitte des 15. Jahrhunderts aufkommenden Printmedien, welche im Zuge ihrer Entstehung zu einer Medialisierung der Kommunikation beigetragen haben - ein Prozess, der auch

52 Merten, Propaganda., S. 154.

53 Ebd., S. 157 und S. 161; Schnurr zeichnet für den Kölner Krieg ein differenzierteres Bild und meint, dass dort dieser Aspekt nicht vorhanden ist, da die Propaganda von unterschiedlichen Stellen ausging: Schnurr, Religionskonflikt, S. 373.

54 Studt, Öffentlichkeit, S. 209; Merten, Propaganda, S. 160-161.

55 Studt, Öffentlichkeit, S. 209.

56 Herbert Marshall MacLuhan/Quentin Fiore, The medium is the massage. Inventory of effects, Harmonsworth 1967.

57 Überblicksmäßig zum Medienbegriff: Würgler, Medien, S. 2.

58 Clemens Zimmermann, Medien, in: EdN, Bd. 8, Stuttgart 2008, Sp. 223-243, hier Sp. 223 und Sp. 240-241; Ein differenzierter Standpunkt: Michael Schilling, Bildpublizistik der frühen Neuzeit. Aufgaben und Leistungen des illustrierten Flugblatts in Deutschland bis um 1700 (Studien und Texte zur Sozialgeschichte der Literatur 29), Tübingen 1990, S. 26. 
deutliche Auswirkungen auf die Politik hatte. ${ }^{59}$ Zuvor manifestierte sich der politischöffentliche Raum vorwiegend am Medium der symbolischen Interaktion. Doch fortan drangen immer mehr technisch neue Medien in diesen Bereich vor und prägten ihn nachhaltig. ${ }^{60}$ Die damit bewirkte Veränderung hatte auch Konsequenzen für die Propaganda der Frühen Neuzeit, die ebenfalls an zeitspezifische Medien gebunden und damit gleichzeitig in ihrer Intensität und Reichweite bestimmt war. ${ }^{61}$

Zahlreiche spätmittelalterliche und frühneuzeitliche Protagonist*innen waren sich der Bedeutung und der Reichweite der aufkommenden Drucktechnik bewusst. Sie verwendeten früh Einblattdrucke, Flugblätter, gedruckte Lieder etc. als Verbreitungsmittel ihrer Absichten. Besonders deutlich wird der Stellenwert dieser neuen Medien während der Reformation. ${ }^{62}$ Doch bereits gut fünfzig Jahre vorher bedienten sie sich der Druckerpresse, um für ihre Sache Stimmung zu machen. Ein prominenter und zugleich relativ früher Fall ist der des Mainzer Erzbischofes Dieter von Isenburg. Dieser stellte sich gegen Kaiser und Papst und versuchte mittels der neuen Medien seine Position darzulegen, die Gegenpartei unrechtmäßiger Handlungen zu bezichtigen und Stimmung für seine Sache im Reich zu machen. ${ }^{63}$ An diesen Zielvorstellungen lassen sich die zuvor erwähnten Strukturmerkmale der Propaganda erkennen. Außerdem erwähnt Studt die weite Verbreitung des von Dieter von Isenburg in Auftrag gegebenen gedruckten Mandates, woraus eine bestimmte Teilöffentlichkeit abgeleitet werden kann. ${ }^{64}$ Falk Eisermann hat indessen darauf aufmerksam gemacht hat, dass es sich beim Mandat von Dieter von Isenburg eher um eine Supplik als um eine frühneuzeitliche Propagandaschrift gehandelt hat, in der ausschließlich mittelalterliche Kommunikationsformen in ein neues Medium transferiert wurden. Dabei geht es um ein gängiges Verfahren der frühen Druckzeit. ${ }^{65}$

Grundsätzlich lässt sich das erwähnte Mandat den Einblattdrucken zuordnen. ${ }^{66}$ Volker Honemann hat für diesen Druck-Typus folgende Erkennungszeichen festgelegt: „a) [...] Papier- oder Pergament-Blatt beliebigen Formats, das in aller Regel nur auf einer Seite beschriftet ist. b) [...] ein vollständiger, in sich abgeschlossener Text (und evtl. zusätzlich ein Bild). c) [...], er existiert also in Gestalt einer Auflage."67 Der erste Punkt behandelt vorwiegend die technische Beschaffenheit des Trägers, welche bewirkte, dass der neuartige einblättrige Träger nicht mehr wie früher so strikt an seinen Entstehungs-

59 Bezogen auf moderne Entwicklungen (elektronische Medien): Winfried Schulze, Politische Kommunikation. Theoretische Ansätze und Ergebnisse empirischer Forschung, Wiesbaden 2011³, S. 13; Frevert, Kommunikation, S. 8.

60 Zimmermann, Medien, Sp. 225-226

61 Studt, Öffentlichkeit, S. 204.

62 Wolfgang Brückner, Flugschrift, in: EdN, Bd. 3, Stuttgart 2006, Sp. 1027-1032, hier Sp. 1028

63 Studt, Öffentlichkeit, S. 225-229.

64 Ebd., S. 229

65 Falk Eisermann, Bevor die Blätter fliegen lernten. Buchdruck, politische Kommunikation und die, Medienrevolution' des 15. Jahrhunderts, in: Karl-Heinz Spieß/Oliver Auge (Hrsg.), Medien der Kommunikation im Mittelalter (Beiträge zur Kommunikationsgeschichte 15), Stuttgart 2003, S. 289-320, hier S. 294.

66 Zur Zuordnung der Flugschriften vor 1500 zu den Einblattdrucken: Brückner, Flugschrift, Sp. 1027.

67 Volker Honemann, Vorformen des Einblattdruckes. Urkunden - Schrifttafeln - Textierte Tafelbilder - Anschläge - Einblatthandschriften, in: Volker Honemann (Hrsg.), Einblattdrucke des 15. und frühen 16. Jahrhunderts. Probleme, Perspektiven, Fallstudien, Tübingen 2000, S. 1-44, hier S. 2. 
ort gebunden, sondern mobiler war. ${ }^{68}$ Bezeichnet werden die Medien dabei oft als „buoch“, „buechlin“, „gemel“, "trucke” oder „zedl”. Der am weitesten verbreitete Terminus ist laut Gisela Ecker „brief“69 In dieser Bezeichnung lassen sich die Fortführungen mittelalterlicher kommunikativer Praktiken erkennen. ${ }^{70}$

Relevant für die Propaganda ist vor allem das zweite formulierte Wesensmerkmal des Einblattdruckes - nämlich der Text. Auch hier sind die Kontinuitätslinien zum Mittelalter deutlich zu erkennen. So kann eine Vielzahl an Textformen für den Einblattdruck konstatiert werden. Hinsichtlich der Fragestellung drängen sich besonders zwei Gestalten auf, nämlich die der Mandate und Polemiken. ${ }^{71} \mathrm{Ob}$ dabei ebenso Analogien der zu analysierenden Texte zu mittelalterlichen Formen bestehen, gilt es in der Quellenbearbeitung zu diskutieren. Erwähnt werden muss hier speziell die Nutzbarmachung dieses neuen Mediums als publizistisches Instrument zur Erreichung bestimmter Kommunikationsziele. ${ }^{72}$ Denn Protagonisten, wie Kaiser Maximilian, waren sich durchaus der Bedeutung dieses neuen Mediums bewusst und setzten es gezielt ein. ${ }^{73}$ Eine letzte Anmerkung betrifft die Auflagenhöhe der Einblattdrucke. Generell bewegten sich diese im Bereich zwischen hundert und tausend Stück. Eisermann legt den Durchschnitt bei etwa dreihundert bis fünfhundert Stück fest und bezieht sich dabei hauptsächlich auf Schätzungen für die Frühdruckzeit. ${ }^{74}$ Genaue Zahlen hingegen sind kaum überliefert. ${ }^{75}$ Dementsprechend ist die genaue Auflagenhöhe kein zwingender Indikator für die Verbreitung eines Schriftgutes, da durch die mündliche Kommunikation die Drucke unabhängig davon eine schwer zu bestimmende Multiplikation erfuhren.

\section{Maximilian I. - Mandat zur Gefolgschaftstreue (22. April 1499)}

Dieses exemplarisch gewählte Mandat Maximilians wird gerne als Beweis für die Absicht herangezogen, das Reich und insbesondere den schwäbischen Adel für den Krieg gegen die Eidgenossen zu mobilisieren. ${ }^{76}$ In dieser Tradition stehen auch die zahlrei-

68 Ebd.

69 Gisela Ecker, Einblattdrucke von den Anfängen bis 1555. Untersuchungen zu einer Publikationsform literarischer Texte (Göppinger Arbeiten zur Germanistik 314), Göppingen 1981, S. 48.

70 Eisermann, Blätter, S. 294.

71 Wolfgang Brückner, Einblattdruck, in: EdN, Bd. 3, Stuttgart 2006, Sp. 118-120, hier Sp. 119.

72 Eisermann, Blätter, S. 299.

73 Stephan Füssel, Der Theuerdank von 1517. Kaiser Maximilian und die Medien seiner Zeit, Eine kulturhistorische Einführung von Stephan Füssel, München 2003, S. 20; Eisermann, Blätter, S. 300.

74 Falk Eisermann, Auflagenhöhe von Einblattdrucken im 15. und frühen 16. Jahrhundert, in: Honemann (Hrsg.), Einblattdrucke des 15. und frühen 16. Jahrhunderts. Probleme, Perspektiven, Fallstudien, Tübingen 2000, S. 143178, hier S. 145-146.

75 Eine hohe Verlustquote ergab sich aus der Weiterverwendung des Beschreibstoffes. Dieser Fall trifft auch für zwei Flugblätter zum Schwabenkrieg zu: Nikolaus Henkel, Ein unveröffentlichtes deutsches Flugblatt Sebastian Brants. Die Klage des Friedens gegen den Krieg und die Verteidigung des Kriegs gegen den Frieden, in: Rudolf Bentzinger (Hrsg.), Grundlagen: Forschungen, Editionen und Materialien zur deutschen Literatur und Sprache des Mittelalters und der Frühen Neuzeit (Zeitschrift für deutsches Altertum und deutsche Literatur, Beiheft 18), Stuttgart 2013, S. 523-534, hier S. 524-525.

76 Bettina Braun, Die Habsburger und die Eidgenossen im späten Mittelalter, in: Irmgard Christa Becker (Hrsg.), Vorderösterreich, nur die Schwanzfeder des Kaiseradlers? Die Habsburger im deutschen Südwesten, Ulm 1999, S. 129-145, hier S. 129; Auch Niederhäuser und Sennhauser betonen die Intention von Maximilian in Bezug auf die Propaganda im Schwabenkrieg: Peter Niederhäuser/Raphael Sennhauser, Kaiser Maximilian I. und die Eidgenossen. Kunst und Propaganda des «letzten Ritters», in: Peter Niederhäuser (Hrsg.), Vom "Freiheitskrieg" zum Geschichtsmythos. 500 Jahre Schweizer- oder Schwabenkrieg, Zürich 2000, S. 73-102, hier S. 82-83. 
chen anderen Mandate, die Maximilian zeit seines Lebens als Medien politischer Kommunikation genutzt hat. ${ }^{77}$ Primär sind Mandate als obrigkeitliche Befehle aufzufassen, die eine weitverbreitete Quellengattung des Mittelalters und der Frühen Neuzeit sind.78 Der Mediävist Alfred Gawlik bringt zur Geltung, dass Mandate in ihrer Form und ihrem Inhalt dem mittelalterlichen Brief ähneln und in ihrer Funktion in erster Linie dazu dienten, Privilegien oder Vergleichbares zu ergänzen. Daher sind sie im Allgemeinen auch relativ schlicht gehalten. ${ }^{79}$

\section{1 Äußere Beschreibung der Quelle}

Überliefert ist uns das gewählte Mandat in mehreren Ausgaben, die in ihren Ausführungen ein wenig voneinander abweichen. Die wohl bekannteste Erwähnung findet sich in der Berner Chronik von Valerius Anshelm. Diese Quelle haben Sieber-Lehmann et. al. in ihre Edition der anti-eidgenössischen Texte aufgenommen. ${ }^{80}$ Interessanterweise zweifelt Niederstätter in Anbetracht dieser Überlieferungssituation die Echtheit des Mandates an. ${ }^{81}$ Anscheinend war Niederstätter unbekannt, dass sich dieses in ähnlicher Weise in Archiven wie dem Stadtarchiv Frankfurt, dem Landesarchiv Sachsen-Anhalt oder dem Stadtarchiv Köln erhalten hat. ${ }^{82}$ Doch auch die Regesta Imperii erwähnen einzig die Tradierung durch Anshelm Valerius. ${ }^{83}$

In Anbetracht einzelner zeitgenössischer Reaktionen und durch Vergleichsquellen ist aber von der Echtheit der Quelle auszugehen. So bezieht sich Willibald Pirckheimer, der Führer des nürnbergischen Kontingents im Schwabenkrieg, auf die Mahnung des

77 Peter Diederichs, Kaiser Maximilian I. als politischer Publizist, Heidelberg 1933, S. 12-17.

78 Heidelberger Akademie der Wissenschaften (Hrsg.), Mandat, in: DRW, Bd. 9, Stuttgart 1996, Sp. 100-103.

79 Alfred Gawlik, Mandat, in: Lexikon des Mittelalters, Bd. 6, Stuttgart 1999, Sp. 186-187, hier Sp. 186.

80 Claudius Sieber-Lehmann/Christian Bertin/Thomas Wilhelmi (Hrsg.), In Helvetios-wider die Kuhschweizer. Fremdund Feindbilder von den Schweizern in antieidgenössischen Texten aus der Zeit von 1386 bis 1532 (Schweizer Texte n.F. 13), Bern 1998, S. 88-91.

81 Alois Niederstätter, Österreich wider den «Erbfeind aller Ritterschaft, allen Adels und aller Ehrbarkeit». Zur Rolle Maximilians I. und seiner Räte im Schwabenkrieg, in: Historischer Verein des Kantons Solothurn (Hrsg.), Gedenkschrift 500 Jahre, S. 133-173, hier S. 157.

82 M2205005, Maximilian I., Römischer Kaiser: Mahnung an die Reichsstände, Hilfe gegen die Eidgenossen und die Graubündner zu leisten, Formular für Fürsten, Freiburg im Breisgau 22. IV. 1499. Staatsbibliothek zu Berlin, Stiftung Preußischer Kulturbesitz, [https://www.gesamtkatalogderwiegendrucke.de/], eingesehen 24. 3. 2019; Mappe Einbl. (Ohly-Sack 1936) - Mahnung an die Reichsstände, Hilfe gegen die Eidgenossen und die Graubündner zu leisten, Freiburg im Breisgau 22. IV. 1499. Universitätsbibliothek Frankfurt am Main, [http://sammlungen. ub.uni-frankfurt.de/inc/urn/urn:nbn:de:hebis:30:2-241789], eingesehen 24. 3. 2019; M22051, Maximilian I., Römischer Kaiser: Mahnung an die Reichsstände, Hilfe gegen die Eidgenossen und die Graubündner zu leisten. Freiburg im Breisgau 22. IV. 1499. Staatsbibliothek zu Berlin, Stiftung Preußischer Kulturbesitz, [https:// www.gesamtkatalogderwiegendrucke.de/pics/22051.jpg], eingesehen 24. 3. 2019; M2205110, Maximilian I., Römischer Kaiser: Mahnung an die Reichsstände, Hilfe gegen die Eidgenossen und die Graubündner zu leisten, Formular für adlige Stände, Freiburg im Breisgau 22. IV. 1499. Staatsbibliothek zu Berlin, Stiftung Preußischer Kulturbesitz, [https://www.gesamtkatalogderwiegendrucke.de/pics/2205110.jpg], eingesehen 24. 3. 2019; M22052, Maximilian I., Römischer Kaiser: Mahnung an die Reichsstände, Hilfe gegen die Eidgenossen und die Graubündner zu leisten, Formular für besondere Fälle, Freiburg im Breisgau 22. IV. 1499. Staatsbibliothek zu Berlin, Stiftung Preußischer Kulturbesitz, [https://www.gesamtkatalogderwiegendrucke.de/pics/22052.jpg], eingesehen 24. 3. 2019.

83 Hermann Wiesflecker/ Christa Beer u. a. (Hrsg.), Regesta Imperii, 1499-1501, Bd. XIV/3 Teil 1: Maximilian I. 1499-1501, Wien-Köln-Weimar 1996, S. 31-32. 
Königs ${ }^{84}$ Wuttke mutmaßt im Folgenden, dass Pirckheimer bereits ein Exemplar des Mandates gesehen haben muss. ${ }^{85}$ Diese Hypothese ist aber kritisch zu betrachten. Denn wenn die Frankfurter Ausgabe berücksichtigt wird, fällt auf, dass vormals ein Siegel angebracht gewesen sein musste. Es könnte deshalb postuliert werden, dass es sich bei der Ausgestaltung dieses Mandates um eine Fortführung des mittelalterlichen litterae clausae handelt. ${ }^{86}$ Damit ergibt sich ein widersprüchliches Verhältnis zwischen der Verbreitung des Inhaltes und der äußeren Beschaffenheit der Quelle, woraus sich nur schwer eine entsprechende Öffentlichkeit ableiten lässt. ${ }^{87}$

Entstanden sein dürfte die Quelle höchstwahrscheinlich in Mainz, wie der Gesamtkatalog der Wiegedrucke zeigt. Hier wird bei den Drucklegungen Peter Schöffer als ausführender Drucker genannt. ${ }^{88}$ Dieser hatte sich bereits beim Streit um die Mainzer Stiftsfehde als Drucker politischer Mandate hervorgetan. ${ }^{89}$ Der Kontakt zu Peter Schöffer ist höchstwahrscheinlich über den Mainzer Erzbischof Berthold von Henneberg herzustellen, der zum damaligen Zeitpunkt Leiter der Reichskanzlei war und somit zuständig für die Aussendung von Schriftgut an die Stände des Alten Reiches. ${ }^{90}$ Der konkrete Druckauftrag dazu ist nicht überliefert.

Über die Datierung gibt die Frankfurter Ausgabe folgenden Hinweis: „[...] an Montag nach Sontag Jubilate Anno dni $\neg$ c. XCIX Vnsers Reichs des Rœemischen Im vierzehenden lare. ${ }^{\prime 91}$ Auch die anderen Einträge im GW zeugen davon, dass die Drucke um diesen Zeitpunkt herum entstanden sein dürften. ${ }^{92}$ Abseits der Drucke wird der entsprechende Zeitraum durch einen Brief von Willibald Pirckheimer verifiziert. Dieser erwähnt das Mandat in einem an die Stadt Nürnberg gerichteten Brief und datiert ihn mit „datum Lindaw auff dinstag nach dem Heyligen Auffartag Anno etc. 99.933 Dabei handelt es sich um den 11. Mai 1499, womit klar sein dürfte, dass zu diesem Zeitpunkt das Mandat

84 „Auch Lieben Herren gend An alle stend deß Reychs Neue manung vnd ernstlich aus, mogen sich eure .w. auch dar nach richten.": Dieter Wuttke, Ein unbekannter Brief von Willibald Pirckheimer, in: Archiv für Kulturgeschichte 50 (1968), S. 294-299, hier S. 299.

85 Ebd.

86 Maximilian I. Römischer König, Mahnung an die Reichsstände, Hilfe gegen die Eidgenossen und die Graubündner zu leisten, Freiburg, 22. IV. 1499 [Mainz, Peter Schöffer, vor 5. V. 1499], Mappe Einblattdrucke 981, Stadt- und Universitätsbibliothek Frankfurt am Main, auffindbar unter Nr. 1936 in: Kurt Ohly/Nera Sack (Hrsg.), Inkunabelkatalog der Stadt- und Universitätsbibliothek und anderer öffentlicher Sammlungen in Frankfurt am Main, Bd. 1, Frankfurt am Main 1967, S. 357; auch unter: Mappe Einbl. (Ohly-Sack 1936) - Mahnung an die Reichsstände, Freiburg im Breisgau 22. IV. 1499; Alfred Gawlik, Litterae, in: Lexikon des Mittelalters, Bd. V, Stuttgart 1999, Sp. 2023-2024, hier Sp. 2023.

87 Im Druck M2205110 wird beispielsweise auf der Rückadresse „Dem Edeln vnnserm vnd des Reiches lieben getrewen Johannsen Grauen zu Nassaw vnd zu Beylstain" angeführt; Im Druck M2205010 steht auf der Rückadresse „Den Ersamen vnnsern vnd des heiligen Reichs lieben getrewen Burgermeister vnd Rate der Stat frannckfort".

88 M2205005, Maximilian I., Formular für Fürsten; Mappe Einbl. (Ohly-Sack 1936) - Mahnung an die Reichsstände; M22051, Maximilian I., Freiburg im Breisgau 22. IV. 1499; M2205110, Maximilian I., Formular für adlige Stände; M22052, Maximilian I., Formular für besondere Fälle.

89 Eisermann, Blätter, S. 293.

90 Leopold Auer, Reichshofkanzlei, in: EdN, Bd. 10, Stuttgart 2009, Sp. 911-914, hier Sp. 912.

91 Mahnung an die Reichsstände, 1499, Einblattdruck 981, UB Frankfurt am Main; Die Abkürzung $\neg$ c bezieht sich auf die mindere Zahl, die durch den Kontext erschlossen wird: Hermann Grotefend, Zeitrechnung des deutschen Mittelalters und der Neuzeit. Glossar und Tafeln, Bd. 1, Hannover 1891, S. 123.

92 Mappe Einbl. (Ohly-Sack 1936) - Mahnung an die Reichsstände, Freiburg im Breisgau 22. IV. 1499.

93 Überliefert bei: Wuttke, Brief, S. 299. 
bereits in Umlauf war. Das im Text letztlich erwähnte Datum bezieht sich höchstwahrscheinlich auf die Abfassung des Schreibens durch die Kanzlei von König Maximilian, der sich zu diesem Zeitpunkt in Freiburg im Breisgau befunden hat, wobei auch dieses Datum bedingt durch die lückenhafte Überlieferung mit Vorsicht zu betrachten ist. ${ }^{94}$

Als Verfasser kommt höchstwahrscheinlich Maximilian I. infrage, der auch in der Intitulatio der Quelle angeführt ist. Bei der Frankfurter Ausgabe steht diese über dem gesamten Schriftblock. ${ }^{95}$ Dennoch ist auch diese Vermutung hinsichtlich der fehlenden Quellen mit einem gewissen Grad an Skepsis in die Untersuchung einzubinden. In früheren Forschungen hat aber schon Peter Diederichs darauf aufmerksam gemacht, dass zumindest von einer ansatzweisen Beteiligung Maximilians bei der Erstellung der Mandate auszugehen ist. ${ }^{96}$ Verstärkend für diese These könnten Werke wie der ${ }_{\text {TT }}$ Theuerdank" angeführt werden. ${ }^{97}$

\subsection{Innere Beschreibung der Quelle}

Zum Inhalt bemerkt Valerius Anshelm nüchtern: „Was der 22. Tag Aprel anno 99; was vil geschrei und wenig woll."98 Diese tendenziöse Äußerung von Anshelm über den Inhalt des Schreibens macht zwar grundsätzlich auf den literarischen Charakter des Mandates aufmerksam, der sich durch zahlreiche Beschimpfungen auszeichnet, reduziert aber gleichzeitig den vollen Bedeutungsumfang. ${ }^{99}$ Maximilian beginnt sein Mandat mit der Betonung, dass nach unzähligen Erlassen gegen die Eidgenossen ihm nichts anderes übrigbleibt, als selbst die Initiative zu ergreifen und in den Krieg zu ziehen. Dabei steckt er seine scheinbaren persönlichen Ziele in Geldern zurück, um mithilfe der Stände des Reiches Widerstand gegen die Eidgenossen zu leisten. Um diesen Widerstand zu legitimieren, führt er die Verfehlungen der Eidgenossen an und meint, dass sie mit ihren Handlungen in erster Linie gegen das Recht ihres eigentlichen Landesherren - des Hauses Österreich - verstoßen haben. Zuvor wird im Mandat erwähnt, dass die Aktionen der Eidgenossen „von der welt unwislich geêret wird"100.

Dementgegen klassifiziert Maximilian die Handlungen als bewusst böswillig, wider den Willen Gottes, ihrer Eide etc. Dann zählt er die zahlreichen Ländereien auf, die sich die Eidgenossen im Laufe der Zeit mit Gewalt einverleibt haben. ${ }^{101}$ Im Anschluss daran

94 Beachte hier die Regesten 9124 und 9125.9124 bezieht sich auf Valerius Anshelm. Der Vergleich mit der Villinger Chronik (9125) darf hier aber nur beschränkt als Beweis verwendet werden, da der originale Wortlaut des Datums fehlt. Vgl. Hermann Wiesflecker/Christa Beer u. a. (Hrsg.), Regesta Imperii, 1499-1501, Bd. XIV/3 Teil 1: Maximilian I. 1499-1501, Wien-Köln-Weimar 1996, S. 31-32.

95 Mahnung an die Reichsstände, 1499, Einblattdruck 981, UB Frankfurt am Main.

96 Diederichs, Maximilian, S. 18-24.

97 Jan-Dirk Müller, Einleitung, in: Jan-Dirk Müller/Hans-Joachim Ziegeler (Hrsg.), Maximilians Ruhmeswerk. Künste und Wissenschaften im Umkreis Kaiser Maximilians I, Berlin 2015, S. 1-6.

98 Valerius Anshelm, Die Berner-Chronik, Bd. 2, herausgegeben von Historischen Verein des Kantons Bern, Bern 1886, S. 182

99 Volker Reinhardt hat unlängst dazu bemerkt, dass die habsburgische Propaganda nur so «[...] strotzte vor Invektiven.» Reinhardt, Schweiz, S. 140.

100 Anshelm, Berner-Chronik, S. 177.

101 Bei der Aufzählung Maximilians tauchen zahlreiche Landschaften auf, die sich vormals im Besitz des Hauses Habsburg befanden, wie beispielsweise: Lentzburg, Kyburg, etc. In der Edition der Berner Chronik wird vermerkt, dass viele der Namen aufgrund ihrer Orthografie nicht klar zugeordnet werden können: Anshelm, BernerChronik., S. 177-179. 
präsentiert Maximilian seine eigene landesherrliche Rolle als passiv und fügt hinzu, dass mit Güte bei den Eidgenossen nichts zu erreichen sei. Anschließend geht er dazu über, sie mit den "grusamen Turken und verspoter unsers kristlichen gloubens [...]." gleichzusetzen, wodurch er sie zum Reichsfeind erhebt. ${ }^{102}$ In diesem Zug spricht das Mandat den Eidgenossen auch jegliche Ehre, Tugend, Mäßigung etc. ab und merkt an, dass sie darum bestraft werden sollten. Schließlich wird der konkrete Kriegsgrund mit den Eidgenossen genannt, nämlich der Angriff aus Graubünden auf die Herren von Brandis. Auch die derzeitige Lage des Krieges wird geschildert, so befinden sich laut Quelle zum Zeitpunkt der Ausstellung zwei Heere der Eidgenossen in der Nähe des Bodensees. ${ }^{103}$

Deshalb hebt Maximilian erneut das Bedürfnis dieses Konfliktes hervor und appelliert an die adressierte Öffentlichkeit, sich mit ihren Streitkräften den Kampfhandlungen anzuschließen. Ein letzter wichtiger inhaltlicher Aspekt bekräftigt die Voraussagung bestimmter Folgen für die Leserschaft dieses Schreibens. Einerseits preist Maximilian sein Wohlwollen im Falle einer Unterstützung an, andererseits deutet er auf die Pflicht des Gehorsams hin, welche die Stände zu erfüllen haben. ${ }^{104}$

\section{Hintergründe und Kontextualisierung}

Aufgrund ihrer Aussagen hat die präsentierte Quelle eine auffällige Rezeption in der Forschungsgeschichte erfahren. Die Standpunkte könnten dabei nicht unterschiedlicher sein. Sie gehen von einer Hinterfragung der tatsächlichen Überlieferung bis hin zur Einschätzung als "gehässige[r] Aufruf zum Reichskrieg". ${ }^{05}$ Trotz der wissenschaftlichen Einschätzung sind vorerst im Abgleich zur gestellten Forschungsfrage der Sender und die adressierte Öffentlichkeit sowie die dazugehörige Interdependenz zu rekonstruieren, um im Anschluss die Quelle entsprechend einordnen zu können. Zu diesem Zweck wird in diesem Kapitel auf drei wesentliche Sachverhalte Rücksicht genommen. Erstens: die Skizzierung des Konfliktfeldes, wobei lediglich die Konfliktkonstellation und damit einhergehend die politischen wie ideologischen Rahmenbedingungen ermittelt werden. ${ }^{106}$ In einem zweiten Schritt werden Maximilian und der Schwäbische Bund als Akteure innerhalb der Kommunikationssituation lokalisiert. Schlussendlich wird noch eine Vergleichsquelle präsentiert, die einen relativen klaren Adressatenkreis aufweist, zumal damit eine profundere Analyse möglich ist.

\footnotetext{
102 Ebd., S. 179.

103 Ebd., S. 181.

104 „Und begerend dem nach abermal an uch, mit allem ernstlichen und hohen fliss, ermanend ouch uch alles des, damit ir uns und dem heiligen rich verwant sind, [...], angesichts diss briefs, tag und nacht ganz ilends uns zůziehen, und uch darin in keinen weg sumig erzœgen. [...] Daran tůnd ir unsern willen und sunder wolgefaleln, mit allen gnaden gegen uch und gmeinen stat zů erkennen und zů gut nimmer vergessen." Anshelm, BernerChronik, S. 182.

105 Niederstätter zweifelte die Echtheit des Mandates an: Niederstätter, Erbfeind, S. 157; Die Einschätzung als Aufruf zum Reichskrieg stammt von: Niederhäuser/Sennhauser, Maximilian I., S. 97.

106 Carl verweist im Zusammenhang der Ideologie auch auf die historiographischen Implikationen, die sich in den folgenden Jahrhunderten niedergeschlagen haben: Horst Carl, «Schwabenkrieg» oder «Schweizerkrieg»? Der Schwäbische Bund als Gegner der Eidgenossenschaft, in: Historischer Verein des Kantons Solothurn (Hrsg.), Gedenkschrift 500 Jahre, S. 97-130, hier S. 99.
} 


\subsection{Konfliktkonstellation des Schwabenkrieges}

Generell ist der Schwabenkrieg anhand seiner regionalen Bedeutung für den süd-deutschen Raum und damit auch für Schwäbisch-Österreich zu charakterisieren. Insbesondere für die Eidgenossen und Habsburg-Österreich gilt dieser Konflikt als einer der blutigsten Auseinandersetzungen ihrer Beziehung. ${ }^{107}$ Seinen Ursprung hat der Krieg dabei in unterschiedlichen kleineren territorialen Spannungen, im ideologischen Gegensatz der Parteien und der Hausmachtpolitik der Habsburger. ${ }^{108}$ Weitere Gründe waren das Söldnerpotential der Eidgenossenschaft, der dazugehörige Kampf um Italien und die Ablehnung der Reichsreform von 1495. ${ }^{109}$ Die drei letztgenannten Faktoren werden allerdings zugunsten der Fokussierung auf die kommunikativen Ziele der Propaganda und den Kontext Vorderösterreich weitestgehend nur angedeutet. Gleichwohl ist jede einzelne dieser Komponenten in ihrer diachronen Dimension zu begreifen, da eine Reduzierung auf das Konfliktjahr 1499 nicht den kontextuellen Umfang widerspiegelt.

Die Habsburger zählten bereits in der zweiten Hälfte des 13. Jahrhunderts zu den führenden Grafengeschlechtern im süddeutschen Raum. ${ }^{110}$ Die Zeit ab dem 14. Jahrhundert war aber von einer gegenteiligen Entwicklung geprägt. So konnte sich die Schweizerische Eidgenossenschaft zusehend als Ordnungsmacht positionieren. Diese Konsolidierung kam in Konflikt mit den Interessen der Habsburger, welche die Länder der Vorlande mit den Österreichischen zusammenführen wollten. Laut Wiesflecker waren diese Bestrebungen vorerst nicht von Erfolg gekrönt. ${ }^{11}$ Maximilian I. verfolgte schließlich eine entschiedenere Politik in Bezug auf die Vorlande. Ihm lag es daran, den südwestdeutschen Raum deutlicher an das Haus Habsburg zu binden. ${ }^{112}$ Zur Durchsetzung ihrer Interessen zogen die Habsburger den Schwäbischen Bund heran, der ursprünglich zur Abwehr der wittelsbachischen Einflussnahme im Südwesten gegründet wurde, sich nun aber immer mehr zu einem ihrer zentralen politischen Instrumente entwickelte. ${ }^{113}$ Die Eidgenossen fühlten sich durch die Aktivitäten des Schwäbischen Bundes zusehends bedrängt. Maximilian forcierte dabei eine informelle Politik, die

107 Niederstätter, Erbfeind, S. 133.

108 In ähnlicher Weise: Alois Niederstätter, Geschichte Vorarlbergs. Vorarlberg im Mittelalter, Bd. 1, Innsbruck 2014, S. 11.

109 Reinhardt, Schweiz, S. 139-140; Meyer betont die Bedeutung der Italienpolitik für den Schwabenkrieg: Meyer, Krisen, S. 23; Die Einführung der Reichsreform 1495 wirkte sich laut Niederstätter ebenfalls nachteilig auf die Beziehung zwischen der Eidgenossenschaft und dem Heiligen Römischen Reich aus: Niederstätter, Erbfeind, S. 138.

110 Heimann, Die Habsburger, S. 24.

111 Hermann Wiesflecker, Österreich im Zeitalter Maximilians I. Die Vereinigung der Länder zum frühmodernen Staat, der Aufstieg zur Weltmacht, Wien 1999, S. 93-194.

112 Bereits relativ früh: Volker Press, Vorderösterreich in der habsburgischen Reichspolitik des späten Mittelalters und der frühen Neuzeit, in: Hans Maier (Hrsg.), Vorderösterreich in der frühen Neuzeit, Sigmaringen 1989, S. 1-42, hier S. 8-9; Franz Quarthal, Die habsburgischen Landstände in Südwestdeutschland, in: Günther Bradler (Hrsg.), Von der Ständeversammlung zum demokratischen Parlament. Die Geschichte der Volksvertretungen in BadenWürttemberg, Stuttgart 1987, S. 79-92, hier S. 83; Laut Metz suchte Maximilian deutlich die Zusammenarbeit mit den Ständen der Vorlande: Axel Metz, Der Stände oberster Herr. Königtum und Landstände im süddeutschen Raum zur Zeit Maximilians I. (Veröffentlichungen der Kommission für Geschichtliche Landeskunde in BadenWürttemberg Reihe B, Forschungen 174), Stuttgart 2009, S. 241; Heimann, Die Habsburger, S. 45.

113 Niederstätter, Geschichte Vorarlbergs, S. 111; Reinhardt, Schweiz, S. 137-138. 
vor allem an die kleineren Stände gerichtet war. ${ }^{114}$ Diese Beeinflussung war aber oft schwierig durchzusetzen, wie Carl klarstellt. ${ }^{115}$

Wie Niederstätter herausgehoben hat, verstärkte ein grundlegender ideologischer Gegensatz das Konfliktpotential, der unter verschiedenen Gesichtspunkten zu betrachten ist. ${ }^{116}$ Zunächst ist auf die habsburgischen Ritterheere zu verweisen, die in Schlachten wie beim Morgarten oder bei Sempach vernichtende Niederlagen erlitten hatten. Stettler spricht dabei von einem „Aderlaß unter dem südwestdt. Adel", der in der Folge den Standpunkt Habsburgs in den Vorlanden deutlich schwächte. ${ }^{117}$ Umso weniger verwunderlich ist es, dass gerade die Erinnerung an diese verlorenen Schlachten bei den Nachfahr*innen der Verlierer negative Konnotationen weckten. ${ }^{18}$ Doch die erwähnten Schlachten hatten nicht nur eine ideologische Wirkung, sondern führten dazu, dass sich langsam eine neue Art der Kriegsführung durchsetzte. Die oberdeutschen Gebiete begannen nun gleich nach den Eidgenossen die sogenannten Landsknechte als Söldner für ihre kriegerischen Unternehmungen einzusetzen. ${ }^{119}$ Daraus ergab sich ein gewisser Berufsneid zwischen „Reisläufern“ und Landsknechten. Anfänglich hegten die Landsknechte noch Sympathien für die eidgenössische Sache. ${ }^{120}$ Im Schwabenkrieg kam es jedoch zu einer Neubewertung der Beziehung, bedingt durch die einsetzende Propaganda der habsburgischen Seite, die fortan die Landsknechte auf einer emotionalen Ebene manipulierte.121 Entgegen der offiziellen Propaganda lassen sich hier hauptsächlich Lieder als zentrale Medien feststellen, die den Konflikt anheizten. ${ }^{122}$ Thematisch verarbeiteten diese vorangegangene Ereignisse des Konfliktes. ${ }^{123}$

In der älteren Literatur ist oft die Rede von geschlossenen Kriegsparteien. ${ }^{124}$ Inzwischen ist diese Ansicht aber der Vorstellung gewichen, dass im Schwabenkrieg zahlreiche latente Spannungen kumulierten. Das Spektrum der anschließend ausbrechenden

114 Volker Press, Die Erblande und das Reich von Albrecht II. bis Karl VI. (1438-1740), in: Robert A. Kann/Friedrich E. Prinz (Hrsg.), Deutschland und Österreich. Ein bilaterales Geschichtsbuch, Wien-München 1980, S. 44-88, hier S. 54; Zur informellen Politik der Habsburger auf die mindermächtigen Stände der Vorlande: Metz, Stände, S. 35.

115 Horst Carl, Der Schwäbische Bund 1488-1534. Landfrieden und Genossenschaft im Übergang vom Spätmittelalter zur Reformation (Schriften zur südwestdeutschen Landeskunde 24), Leinfelden 2000, S. 503.

116 Niederstätter, Geschichte Vorarlbergs, S. 111-112.

117 Bernhard Stettler, Sempach, Schlacht bei, in: Lexikon des Mittelalters, Bd. VII, Sp. 1742.

118 Steffen Krieb, Vom Totengedenken zum politischen Argument. Die Schlacht bei Sempach (1386) im Gedächtnis des Hauses Habsburg und des südwestdeutschen Adels im 15. Jahrhundert, in: Dieter Langewiesche/Friedrich Lenger u. a. (Hrsg.), Kriegsniederlagen. Erfahrung und Erinnerung, Berlin 2015, S. 69-88, hier S. 80-87.

119 Matthias Rogg, Landsknecht, in: EdN, Bd. 7, Stuttgart 2008, Sp. 559-561, hier Sp. 559.

120 Sonja Kerth, Der landsfrid ist zerbrochen. Das Bild des Krieges in den politischen Ereignisdichtungen des 13. bis 16. Jahrhunderts (Imagines medii aevi 1), Wiesbaden 1997, S. 64-65; Auch Meyer unterstreicht die Sympathie für die Eidgenossenschaft, konkretisiert aber den beschriebenen Personenkreis nicht: Meyer, Krisen, S. 29.

121 Claudius Sieber-Lehmann, Einleitung, in: Claudius Sieber-Lehmann/Christian Bertin/Thomas Wilhelmi (Hrsg.), In Helvetios-wider die Kuhschweizer. Fremd- und Feindbilder von den Schweizern in antieidgenössischen Texten aus der Zeit von 1386 bis 1532 (Schweizer Texte n.F. 13), Bern 1998, S. 1-22, hier S. 7-9.

122 Carl, Schwabenkrieg, S. 118-119.

123 Bspw: Anonymus, Spottlied der Landsknechte gegen die Eidgenossen. Januar 1499, in: Sieber-Lehmann/ Bertin/Wilhelmi (Hrsg.), In Helvetios-wider die Kuhschweizer. Fremd- und Feindbilder von den Schweizern in antieidgenössischen Texten aus der Zeit von 1386 bis 1532 (Schweizer Texte n.F. 13), Bern 1998, S. 85-87, hier S. 85 .

124 Mommsen hat schon relativ früh auf diesen Missstand aufmerksam gemacht: Karl Mommsen, Eidgenossen, Kaiser und Reich. Studien zur Stellung der Eidgenossenschaft innerhalb des Heiligen Römischen Reiches (Basler Beiträge zur Geschichtswissenschaft 72), Stuttgart-Basel 1958, S. 234-235. 
Auseinandersetzungen reichte von regionalen Feindseligkeiten, wie dem Kampf um das Münstertal, bis hin zur Position der Städte Rottweil und Konstanz betreffend die Eidgenossenschaft. ${ }^{125}$ Denn wie Braun zur Geltung gebracht hat, bedeutete der Schwabenkrieg die endgültige Klärung zahlreicher Territorialkonflikte. ${ }^{26}$

\subsection{Akteure der Kommunikation}

\subsubsection{Maximilian I.}

Generell wird die Rolle Maximilians im Rahmen des Schwabenkrieges als relativ passiv bewertet. Noch während der ersten Kampfhandlungen befand er sich in Geldern. Diese Tatsache nimmt Niederstätter zum Anlass, das geringe Interesse Maximilians an einer kriegerischen Eskalation zwischen den Eidgenossen und Habsburg zu erklären. Vielmehr werden die tirolerischen Stände als Kriegstreiber dargestellt, zumal diese für das Ausgreifen nach Graubünden verantwortlich gemacht werden. ${ }^{127}$ Die Untätigkeit Maximilians wird oft dahingehend gedeutet, dass er die Absicht verfolgte, über die Söldnerheere der Eidgenossen verfügen zu können. ${ }^{128}$ Auch Manfred Hollegger erkennt im Vorgehen Maximilians eine ähnliche Intention. Für ihn liegt auf der Hand, dass vor allem der Schwäbische Bund auf einen Krieg mit den Eidgenossen drängte, allen voran der bundesverwandte Mainzer Erzbischof Berthold von Henneberg. ${ }^{129}$

Dabei agierte Maximilian als Herr der Vorlande relativ bedacht. So änderte er die Politik seines Vaters, der den Großteil seiner Regierungszeit in Innerösterreich verweilte, indem er seine Präsenz in den Vorlanden deutlich verstärkte. ${ }^{130}$ Diese deutlichere Gegenwart in den Vorlanden könnte durchaus ein Indiz für die versuchte Ausweitung des habsburgischen Einflusses sein. ${ }^{131}$ Die Tatsache, dass Maximilian schon zuvor zweimal bei Schlichtungsverfahren zwischen Bayern und dem Schwäbischen Bund extra aus den Niederlanden anreiste, belegt sein Interesse am süddeutschen Raum. ${ }^{132}$

Daneben dürfte die Ausdehnung der habsburgischen Herrschaft in den Vorlanden gute Gründe gehabt haben: Durch eine verstärkte Einflussnahme hätten die Städte dichter an das Haus Habsburg gebunden und mittels größeren Besitzungen im schwä-

125 Zum Kriegsausbruch im Münstertal: Niederstätter, Erbfeind, S. 139-145; Zur Rolle der Städte Rottweil und Konstanz: Reinhardt, Schweiz, S. 139-140; Winfried Hecht, Rottweil ca. 1340-1529. Im Herbst des Mittelalters, Rottweil 2005, S. 121-124; Zum Oberrhein als Konfliktgebiet: Speck, Vorderösterreichs, S. 107-109. Braun, Die Habsburger, S. 129-130.

127 Niederstätter, Erbfeind, S. 139-145.

128 Braun, Die Habsburger, S. 129-130 und S. 144; Niederstätter, Erbfeind, S. 135.

129 Manfred Hollegger, Maximilian I. Herrscher und Mensch einer Zeitenwende, Stuttgart 2005, S. 106-107.

130 Press, Vorderösterreich, S. 10; Müller, Einleitung, S. 1.

131 Mitunter auch durch die Einführung eines Landsassiats: Wolfgang Wüst, Die Adelskurien. Zwischen vorderösterreichischer Landsässigkeit, ständischer Autonomie und Reichsfreiheit: Die »Insassen« in der Markgrafschaft Burgau, die »Anstösser« in der Landvogtei Schwaben und die Hegauer Reichsritter in der Landgrafschaft Nellenburg, Ein Vergleich, in: Becker (Hrsg.), Vorderösterreich, nur die Schwanzfeder des Kaiseradlers? Die Habsburger im deutschen Südwesten, Ulm 1999, S. 188-195, hier S. 191; Quarthal, Landstände, S. 83.

132 Reinhard Stauber, Maximilian I. als ,politischer Virtuose', in: Müller/Ziegeler (Hrsg.), Maximilians Ruhmeswerk. Künste und Wissenschaften im Umkreis Kaiser Maximilians I., Berlin 2015, S. 7-30, hier S. 16. 
bischen Raum die Legitimität des Königtums unterstrichen werden können. ${ }^{133}$ Denn Schwaben galt traditionellerweise als Gebiet der Könige. ${ }^{134}$

Zwei weitere Faktoren betreffend die Position Maximilians dürften einmal die Gefahren für das Reichsgebilde im Ganzen und andererseits der Verdruss über profranzösische Haltung der Eidgenossen gewesen sein. ${ }^{135}$ Gerade Ersteres steht in einem Spannungsverhältnis zur Selbstwahrnehmung Maximilians, dessen Bild vom Ideal des mittelalterlichen Kaisertums geprägt war. In dieser Situation hatte er es sich zur Aufgabe gemacht im göttlichen Auftrag das Alte Reich wiederherzustellen, die Ungläubigen zu bekehren und den Weltfrieden zu sichern. ${ }^{136}$ Dem entgegen positionierten sich die Eidgenossen als Brecher dieses Friedens und als Widerstand gegen die Reichsreform. ${ }^{137}$ Der zweite Punkt reflektiert die Auseinandersetzung um Italien, die ab 1500 die Aufmerksamkeit Maximilians immer mehr bündelte. Insofern meinen Sennhauser und Niederhäuser, dass Maximilian mehr auf dem Papier Krieg gegen die Eidgenossen führte. Die Autoren erkennen daher eine Verlagerung der Aufmerksamkeit weg von der Eidgenossenschaft hin zu Flandern, Burgund, Ungarn etc., wobei Südwestdeutschland immer noch und auch nach 1500 eine wichtige Rolle spielte. ${ }^{138}$

\subsubsection{Vorderösterreich und der Schwäbische Bund}

Um im Wesentlichen auf die Vorlande aus kommunikativer Sicht Einfluss zu nehmen, bediente sich Maximilian, wie bereits seine Vorfahren, diverser Bundsysteme, die im schwäbischen Raum eine lange Tradition aufweisen. Diese Zusammenschließungen ermöglichten Meinrad Schaab zufolge für den Landesfürsten die Chance der Territorialisierung. ${ }^{139}$ Für den Kontext des Schwabenkrieges nimmt sich insbesondere der Schwäbische Bund als zentraler Adressat der habsburgischen Propaganda heraus. Das 1488 gegründete Bündnis, welches zeitweise über 570 Mitglieder hatte, wird in jüngst erschienenen Publikationen immer noch wegen seiner Wirkung für den Föderalismus gelobt. ${ }^{140}$ Albert Funk beispielsweise bezeichnet den Schwäbischen Bund, bezogen

133 Gerade für Freiburg ist eine enge Beziehung zu Kaiser Maximilian festzustellen: Hans Schadek, Der Kaiser und seine Stadt. Maximilian I. und seine Beziehung zu Freiburg, in: Hans Schadek (Hrsg.), Der Kaiser in seiner Stadt. Maximilian I. und der Reichstag zu Freiburg 1498 (Zeitschrift des Breisgau-Geschichtsvereins Schau-ins-Land 117), Freiburg im Breisgau 1998, S. 216-273.

134 Press, Vorderösterreich, S. 8-9.

135 Niederhäuser/Sennhauser, Maximilian I., S. 90-92; Zum Verhältnis der Eidgenossen zu Frankreich: Meyer, Krisen, S. 21.

136 Niederhäuser/Sennhauser, Maximilian I., S. 78-79.

137 Reinhardt meint, dass die eingeführten Reformen nicht der politischen Realität der Eidgenossen entsprachen: Reinhardt, Schweiz, S. 138; Meyer schwächt die Rolle der Reichsreform ab und betont, dass dieser Faktor vor allem in der älteren Forschungsliteratur zur Geltung kommt: Meyer, Krisen, S. 18-19.

138 Niederhäuser/Sennhauser, Maximilian I., S. 78.

139 Meinrad Schaab, Spätmittelalter (1250-1500), in: Meinrad Schaab/Hansmartin Schwarzmaier (Hrsg.), Allgemeine Geschichte. Teil 2,Vom Spätmittelalter bis zum Ende des Alten Reiches (Handbuch der baden-württembergischen Geschichte 1), Stuttgart 2000, S. 1-144, hier S. 34-37; Press hat bereits früh auf die Integrationskraft der Bünde aufmerksam gemacht: Press, Die Erblande und das Reich, S. 54.

140 Schaab, Spätmittelalter, S. 37; Zu den Mitgliedern des Schwäbischen Bunde: Ernst Bock, Der Schwäbische Bund und seine Verfassungen. 1488-1534, Ein Beitrag zur Geschichte der Zeit der Reichsreform (Untersuchungen zur deutschen Staats- und Rechtsgeschichte A.F. 137), Aalen $1968^{2}$. 
auf die Durchsetzung einer „bundestaatlichen Organisation" gar als „Erfolgsmodell“.141 Wobei aber zu betonen ist, dass das Bild eines einheitlichen Bundes an der Realität vorbeiführt. ${ }^{142}$ Aufbauend auf zahlreiche vorangegangene Bündnissysteme gelang es dem Bund letztendlich, fast alle Kräfte im schwäbischen Raum zu vereinen. ${ }^{143}$ Einzig Städte wie Rottweil, Konstanz und Buchhorn (Friedrichshafen) blieben dem Bund zunächst fern, aus Respekt gegenüber ihren südlichen Nachbarn - den Eidgenossen. ${ }^{144}$

Der Schwäbische Bund bestand vorwiegend aus einer prohabsburgischen Klientel, hatte jedoch auch Gegner der Reichsreform von 1495 als Mitglieder. ${ }^{145}$ Umso interessanter ist deshalb die Tatsache, dass sich die Begeisterung für die Kriegsteilnahme beim Schwäbischen Bund anfänglich in Grenzen hielt. Dieser Umstand ist an der nicht vollen Ausschöpfung des militärischen Potenzials erkennbar. ${ }^{146}$ Der fehlende Reiz, am Kampf teilnehmen zu wollen, könnte bis zu einem gewissen Grad in den Sympathien für die eidgenössische Sache verortet werden. ${ }^{147}$ Karl Mommsen hat schon früher auf diesen Sachverhalt hingedeutet. ${ }^{148}$ Die aufgestellte These wird jedoch in der neueren Forschungsliteratur bis auf Meyer kaum rezipiert. Insofern ist sie mit besonderer Vorsicht zu betrachten. Auch betont Carl, dass aufgrund politischer Differenzen von keiner allzu großen Identifikation mit den Habsburgern auszugehen ist. ${ }^{149}$

\subsection{Das Mandat Maximilians im Vergleich}

Aufschluss über den relativ unklar umrissenen Adressatenkreis kann die ergänzende Anführung eines weiteren Mandates bieten, das sich durch die direkte Adressierung an den Schwäbischen Bund auszeichnet. Überliefert ist dieses Mandat bei Klüpfel, der das Senkenberg'sche Bundbuch als Ursprung des Schreibens anführt. ${ }^{150}$ Leider konnte diese primäre Quelle nicht lokalisiert werden, wodurch sich die folgenden Ausführungen auf die Edition von Klüpfel beschränken müssen. Hinzu kommt, dass sich in den Regesta Imperii kein Vermerk zur Ausstellung des besagten Schreibens finden lässt. Dementsprechend ist die Quelle äußerst kritisch zu betrachten. ${ }^{151}$

141 Albert Funk, Kleine Geschichte des Föderalismus. Vom Fürstenbund zur Bundesrepublik, Paderborn 2012², S. 7677; Horst Carl, Landfrieden als Konzept und Realität kollektiver Sicherheit im Heiligen Römischen Reich, in: Gisela Naegle (Hrsg.), Frieden schaffen und sich verteidigen im Spätmittelalter. Faire la paix et se défendre à la fin du Moyen Âge (Pariser Historische Studien 98), München 2012, S. 121-138, hier S. 127-130; Press, Vorderösterreich, S. 10.

142 Peter-Johannes Schuler, Schwäbischer Bund, in: Lexikon des Mittelalters, Bd. VII, Sp. 1607-1608, hier Sp. 1607.

143 Der Einfluss der Habsburger ist schon für den Bund vom St. Jörgenschild nachweisbar: Speck, Vorderösterreichs, S. 101.

144 Schaab, Spätmittelalter, S. 37.

145 Carl, Landfrieden, S. 127.

146 Carl, Schwabenkrieg, S. 121-123; Niederstätter, Erbfeind, S. 151.

147 Meyer, Krisen, S. 29

148 Mommsen, Eidgenossen, S. 283; Mommsen stützt seine These dabei auf folgende Quellen: Urkunden zur Geschichte des Schwäbischen Bundes (1488-1533), herausgegeben von Karl Klüpfel, Stuttgart 1846, S. 280, S. 294, S. 319, S. 325, S. 345, S. 346, S. 328 und S. 373.

149 Carl, Schwabenkrieg, S. 107.

150 Maximilian I. Römischer König, Mandat dasz dem pund zugetzogen werden soll. 23. April 1499, in: Klüpfel, Urkunden zur Geschichte des Schwäbischen Bundes (1488-1533). Erster Theil, 1488-1506 (Bibliothek des Literarischen Vereins in Stuttgart 14), Stuttgart 1846, S. 321-322.

151 Dennoch wird die Quelle herangezogen: Patrick Honecker, Vorreformatorische Schlagwörter. Spiegel politischer, religiöser und sozialer Konflikte in der frühen Neuzeit, phil. Diss Trier 2002, S. 11. 
Hierdurch fällt der Aspekt der äußeren Quellenkritik weitestgehend weg. Auch der Zeitpunkt der Erstellung und Ort sind textimmanent zu bestimmen, wobei hinsichtlich der fehlenden Vergleichsquellen die angegebenen Daten bis auf Weiteres zu verwenden sind. Genannt wird somit der 23. April 1499 als Ausstellungsdatum und Mainz als Entstehungsort. ${ }^{152}$ Der Entstehungsort und die im Schluss genannte Formel "Ad Mandatum Domini regis in consilio" erschweren die Bestimmung des Autors. Zwar wird in der Intitulatio Maximilian angeführt, inwiefern er für den Inhalt im Konkreten verantwortlich war, lässt sich aber nur schwer sagen. Denn die erwähnte Formel verweist auf die Reichshofkanzlei, die zum damaligen Zeitpunkt dem Erzbischof von Mainz unterstand und somit die Ausstellung dieses Schreiben wohl unter seine Zuständigkeit fällt. ${ }^{153}$ Berthold von Henneberg wird in der Sekundärliteratur gerne als „unabhängiger Gestalter" der Reichspolitik oder gar als konfliktbereiter Gegenspieler Maximilians beschrieben. ${ }^{154}$ Textimmanente Formulierungen legen aber den Schluss nahe, dass Maximilian der Autor gewesen sein könnte. Eine objektive Bewertung der Quelle ist folglich in so kurzer Form schwer möglich, weshalb sie, ungeachtet der schwierigen äußerlichen Beschreibung und der kaum transparenten Herkunft der Quelle hauptsächlich zur Illustration der angesprochenen Öffentlichkeit dienen soll.

So spricht die Quelle in der Inscriptio direkt die Stadt Augsburg an. Wiederum lassen sich hier Formalia der mittelalterlichen Litterae erkennen. ${ }^{155}$ Der erste Teil des Schreibens ähnelt im Inhalt deutlich dem bereits angeführten Mandat vom 22. April. Insofern wird beschrieben, dass die Eidgenossen gegen das Recht verstoßen und den Landfrieden gebrochen haben, wodurch die erblichen Besitzungen des Hauses Habsburg in Gefahr sind. Entgegen des zuerst beschriebenen Mandates verzichtet der Autor hier auf eine ausführliche Diffamierung der Eidgenossen, sondern stellt die Forderung an die Stadt Augsburg ins Zentrum des Schreibens: „und unserer vorausgegangenen mandaten begryffen, und vermeydung unserer und des reichs schwären ungnad und straffe, von römischer künigclicher macht ernstlich gebietende, und wollen, das jr angesichts ditz briefs zwaintzig zu ross [...] zuschickt"156.

Interessanterweise wird das vorangegangene Mandat explizit erwähnt. Zuletzt werden vergleichbare Aussagen zu den Folgen der Nicht-/Beachtung des Mandates gemacht. Der Verweis auf das vorangegangene Schreiben birgt somit die nachstehenden Implikationen: Das Schreiben könnte durchaus in Abstimmung mit dem König abgefasst worden sein, die zeitliche Nähe verifiziert die Existenz des Schreibens vom 22. April 1499 und nachdrücklich ergingen Schreiben an bestimmte süddeutsche Städte.

152 Maximilian I. Römischer König, Mandat. 23. April 1499, S. 322.

153 Das zedler'sche Universallexikon vermerkt hierzu: "Die von solchem Reichs-Regiment ausgefertige Befehle solten nicht weniger gelten, als die Schlüsse eines Reichstages, und zwar pflegten sie folgende Unterschrift zu haben: ad mandatum Domini Regis in Consilio Imperii, worzu noch ein Churfürst, von welchen allezeit einer um den andern ein viertel jahr in Person dem Reichs-Regiment beyzuwohnen bewiligt hatte, [...]." Reichs-Regiment, in: Johann Heinrich Zedler (Hrsg.), Grosses vollständiges Universal Lexicon Aller Wissenschafften und Künste, Bd. 31 , Halle-Leipzig 1742, Sp. 163-165, hier Sp. 164.

154 Barbara Stollberg-Rilinger, Das Heilige Römische Reich Deutscher Nation, München 20135, S. 28; Funk, Förderalismus, S. 80; Auer, Reichshofkanzlei, S. 912.

155 Gawlik, Mandat, Sp. 186-187; Gawlik, Litterae, Sp. 2023-2024.

156 Maximilian I. Römischer König, Mandat. 23. April 1499, S. 321-322. 


\section{Interpretation und Bewertung}

Wie erwähnt wurde, gestaltet sich die Überlieferung der gewählten Quelle äußerst komplex. Folglich fällt die hier versuchte Bewertung skizzenhaft aus. Festgehalten werden kann jedoch, dass die Quelle der Form nach deutlich in die Tradition des mittelalterlichen Briefes fällt. Diese Beobachtung lässt sich textimmanent ermitteln „in angesichts, dass briefs, [...]". ${ }^{157}$ Unter Hinzunahme der Frankfurter Ausgabe können somit zwei von drei Kriterien von Honemann fixiert werden. ${ }^{158}$ Die Quelle ist als Einblattdruck in Papierform tradiert und beinhaltet einen in sich abgeschlossenen Text. Die Auflage des Dokumentes lässt sich leider nicht mehr feststellen. Zwar erwähnt Eisermann, dass Maximilian I. 1518 einen Befehl an Konrad Peutinger ergehen ließ, mit der Anordnung 300 Exemplare eines Achtmandates drucken zu lassen. ${ }^{159}$ Angesichts der Mitglieder des Schwäbischen Bundes könnte über eine mutmaßliche Druckanzahl gerätselt werden, was aber nicht wirklich zielführend wäre. Somit muss auf den von Eisermann genannten Richtwert für Drucke vor 1500 von ca. 300-500 Stück verwiesen werden. ${ }^{160}$ Dieses Defizit erschwert eine exakte Bestimmung der adressierten Öffentlichkeit, bedeutet aber nicht, dass die Quelle keine adäquate Verbreitung gefunden hat.

Zuvor sollte jedoch eine primäre Annahme bekräftigt werden, die eingangs gestellt wurde. Nämlich, inwiefern das gewählte Medium dem Wesen der Propaganda entspricht. In dieser Hinsicht kann konstatiert werden, dass die gewählte Quelle in ihrer Betrachtung vorläufig alle fünf aufgestellten Wesensmerkmale der Propaganda erfüllt. Deutlich wird hervorgehoben, dass Maximilian der Geschädigte in diesem Konflikt ist. ${ }^{161}$ Verstärkt wird diese Beobachtung durch die Aufzählung der verlorenen Besitzungen. Auch der Zusatz, dass Maximilian eigens für den Krieg seine persönlichen Ziele zurücksteckt, entspricht den Merkmalen von Propaganda. Im Weiteren erfolgt die deutliche Diffamierung der Eidgenossen, in der von "den bòsen, groben und schnöden gepurslüten" die Rede ist, die Maximilian mit den Feinden der Christenheit (Türken) gleichsetzt und gleichzeitig als Gegner der ständischen Ordnung bezichtigt. ${ }^{162}$ Hieraus könnte die erhöhte Bereitschaft des Adels - als Vertreter der ständischen Ordnung gegen die Eidgenossen in den Krieg zu ziehen, geschlossen werden. ${ }^{163}$

Über den Ausschließungscharakter werden im Mandat Maximilians auch die entsprechenden Folgen konstruiert. So meint der König, dass im Falle einer fehlenden Strenge gegenüber den Eidgenossen der ganze Reichsverband in Gefahr sei, weshalb er an die Stände appelliert ihre Heere dem Habsburgischen anzuschließen. ${ }^{164}$ Das Resultat der

157 Anshelm, Berner-Chronik, S. 182.

158 Honemann, Vorformen, S. 2.

159 Eisermann, Auflagenhöhe, S. 170

160 Ebd., S. 145-146.

161 "Darzů wir und wiland unsere vofaren loblicher gedåchtnüss bisshar zů|gesehen und das geliten, und ja wol vil bersuocht, aber nüt geschaft, wider si nichts gehandlet, sunder verhoft haben, mit der zit. mit gủetikeit ichts zu erlangen.“ Vgl. Anshelm, Berner-Chronik, S. 177-179.

162 Anshelm, Berner-Chronik, S. 180; Krieb, Vom Totengedenken, S. 86.

163 Grundlegend zur sozialen Ordnung: Marian Füssel/Thomas Weller, Einleitung, in: Marian Füssel/Thomas Weller (Hrsg.), Ordnung und Distinktion. Praktiken sozialer Repräsentation in der ständischen Gesellschaft (Symbolische Kommunikation und gesellschaftliche Wertesysteme 8), Münster 2005, S. 9-22.

164 Anshelm, Berner-Chronik, S. 181-182. 
Beteiligung ist das Wohlwollen des Königs. Eine Nichtbefolgung des Mandates wäre mit einem Verrat am Reichsverband gleichzusetzen, weshalb hier von einer autoritären Konnotation gesprochen werden kann. Dementsprechend ist vorerst in Bezug auf die strukturellen Merkmale von Propaganda die Rede.

Eine Bestimmung der wesentlichen Elemente der Kommunikation fällt vorerst eindeutig aus. Als Sender tritt Maximilian bzw. die königliche Partei auf, welche die bereits skizzierte Botschaft an einen relativ unscharf umrissenen Empfängerkreis über das Medium des Mandates richtet, was idealerweise in einer Wirkung mündet. ${ }^{165}$ Doch die Bestimmung der angedachten Kommunikation ist weit mehr als die reine Zuordnung der zuvor erwähnten Merkmale. Wie eingangs herausgestellt wurde, handelt es sich bei Kommunikation um die gemeinsame Aktualisierung von Sinn. ${ }^{166}$ Innerhalb der Propaganda erfolgt diese Aktualisierung über die Manipulation der Darstellungen und anschließende Übernahme einer Verhaltensprämisse - meistens determiniert durch die Macht des Senders. ${ }^{167}$ Erkennbar wird dieser Prozess an der anschließenden Reaktion auf die Botschaft des Senders. Die Sinnstrukturen beider Akteure wurden im vorangegangenen Kapitel aufgezeigt. Dabei ist deutlich geworden, dass Maximilian darauf bedacht war, die Stellung seines Hauses in den Vorlanden auszubauen, wobei er bewusst an die ideologischen Gegensätze zwischen den Schwaben und Eidgenossen anknüpfte. Dies erreicht er durch eine Erhebung der Eidgenossen zu Reichsfeinden und die Wachhaltung der Erinnerung an die verlorenen Schlachten der schwäbischen Ritterheere. ${ }^{168}$ Dieser Sinnhorizont entspricht auch bis zu einem gewissen Grad den Vorstellungen der süddeutschen Stände, zumindest was die prohabsburgische Klientel im Schwäbischen Bund betrifft. Auf die ideologischen Abstufungen innerhalb des Bundes wurde bereits hingewiesen. Sichtbar wird dies auch an der fehlenden Bereitschaft der Stände am Konflikt teilzunehmen. ${ }^{169}$ Die Propaganda von Maximilian setzte deshalb genau hier an. Zu diesem Zweck wandte er sich an eine gewisse Teilöffentlichkeit, die in ihren Handlungen und Meinungen manipuliert werden sollte, um vermehrt am Konflikt teilzunehmen.

Mit "Irr lieben, getrüwen!" beginnt im Mandat der eigentliche Text. Diese diffuse Formulierung lässt kaum Schlüsse auf die gewählte Teilöffentlichkeit zu, welche die Quelle zu berühren versuchte. ${ }^{170}$ Zwar versucht die politische Kommunikation tendenziell eine künstliche Öffentlichkeit herzustellen, was sie auch durch die Mechanismen der neuen Medien erreicht haben dürfte. Dieser Prozess ist jedoch schwierig zu bestimmen, zumal hierfür die Quellen fehlen. Die Sekundärliteratur beschreibt zugegebenermaßen, dass Maximilian gezielt die Verbreitung von amtlichen Schreiben forciert hat, dies aber vor allem für die Liederdichtung zutrifft. ${ }^{171}$ Die durchaus öffentliche Verbreitung von politischen Inhalten lässt sich hingegen am Krieg gegen Venedig Anfang des 16. Jahr-

\footnotetext{
165 Beck, Kommunikation, S. 155.

166 Schnurr, Religionskonflikt, S. 41; Luhmann, Soziale Systeme, S. 194 und S. 203.

167 Merten, Propaganda, S. 157.

168 Anshelm, Berner-Chronik, S. 180.

169 Reinhardt, Schweiz, S. 140-141.

170 Anshelm, Berner-Chronik, S. 175.

171 Studt, Öffentlichkeit, S. 232.
} 
hunderts relativ gut belegen. ${ }^{172}$ Jan Dirk Müller fügt hier an, dass sich die öffentlichen Schreiben Maximilians oft an einen diffusen Personenkreis wandten und es daher nur schwer abzuschätzen ist, wer nun mit diesen Schreiben gemeint war. ${ }^{173}$ Abhilfe kann auf zweierlei Arten verschafft werden: zum einen durch Vergleichsquellen und Reaktionen von Zeitgenoss*innen, die aber nur spärlich vorhanden sind. Zum anderen durch die Hinzunahme der äußerlichen Beschaffenheit in die Analyse. Letzteres ist an der Adressierung der Schreiben zu beobachten, die sich an eine dezidierte Teilöffentlichkeit richten, nämlich die adeligen Stände und Städte des Reiches. ${ }^{174}$ Demnach könnte angenommen werden, dass es sich beim Mandat in erster Linie um eine Weisung im Kontext der königlichen Herrschaft gehandelt hat und damit an das gesamte Reich gerichtet war. ${ }^{175}$

Außerhalb von Süddeutschland war das Interesse an dieser Auseinandersetzung relativ gering, weshalb zwar ein Reichskrieg propagiert wurde, der in der Realität aber nur einzelne Stände betraf, die sich in räumlicher Nähe dazu befanden. ${ }^{176}$ Das zweite Mandat, das „dem pund zugetzogen werden soll“, lässt schon konkretere Schlüsse auf den Adressatenkreis zu. ${ }^{177}$ Die deutliche Nennung des Bundes und die anschließende Erwähnung von Augsburg als Ort der Bundeskanzlei erwecken den Eindruck, dass dem Schwäbischen Bund eine besondere Rolle als Adressatenkreis zufällt. ${ }^{178}$ Bestätigung findet diese Annahme im Echo der Bundgenossen, woraus eine entsprechende Teilöffentlichkeit rekonstruiert werden kann. Willibald Pirckheimer schreibt etwa am 11. Mai an die Stadt Nürnberg mit der Bemerkung, dass ein Mandat ausgegangen ist. ${ }^{179}$ Inwiefern Pirckheimer den genauen Inhalt des Mandates gekannt hat, kann aber nicht geklärt werden. Diese Annahme steht im Kontrast zur versiegelten Frankfurter Ausgabe. Nichtsdestotrotz verweist die Bemerkung Pirckheimers auf die Wahrnehmbarkeit der Quelle.

Daneben sind auch andere Reaktionen auf die Mandate Maximilians überliefert: Die Stadt Augsburg geht beispielsweise auf das direkt an sie gerichtete Mandat ein. ${ }^{180}$ Auch die Stadt Nürnberg vermerkt in ihrer Chronik das Ankommen einer Mahnung, wobei

172 Füssel, Theuerdank, S. 22; Jan-Dirk Müller, Publizistik unter Maximilian I. Zwischen Buchdruck und mündlicher Verkündigung, in: Frevert/Braungart (Hrsg.), Sprachen des Politischen. Medien und Medialität in der Geschichte, Göttingen 2004, S. 95-122.

173 Müller, Publizistik, S. 98.

174 Das Mandat spricht hier davon: «das alles haben wir uch, ouch andren unsern und des richsstånden unverkint nit wollen lassen, [...].» Anshelm, Berner-Chronik, S. 182; Vgl. M2205005, Maximilian I., Formular für Fürsten; Mappe Einbl. (Ohly-Sack 1936) - Mahnung an die Reichsstände; M22051, Maximilian I., Freiburg im Breisgau 22. IV. 1499; M2205110, Maximilian I., Formular für adlige Stände; M22052, Maximilian I., Formular für besondere Fälle.

175 Gawlik, Mandat, Sp. 186.

176 Niederhäuser/Sennhauser, Maximilian I., S. 74.

177 Maximilian I. Römischer König, Mandat. 23. April 1499, S. 321.

178 Carl, Schwäbischer Bund, S. 341; Augsburg erhält das Mandat auch: Klüpfel, Urkunden zur Geschichte des Schwäbischen, S. 336.

179 Wuttke, Brief, S. 299.

180 Klüpfel, Urkunden zur Geschichte des Schwäbischen, S. 336-337. 
auch hier auf das Schreiben von Pirckheimer Bezug genommen wird. ${ }^{181}$ Trotzdem kann die exemplarische Anführung dieser Städte nicht als allzu konkreter Beweis für die Adressierung des süddeutschen Raumes betrachtet werden. Als Bestätigung hingegen könnte die in der Sekundärliteratur erwähnte Stärkung der Hausmachtpolitik gesehen werden, die darauf abzielte die Stände Süddeutschlands stärker an den König bzw. an Maximilian in der Rolle als Landesherr zu binden. ${ }^{182}$ Gerade in Schwaben war während schwerer Krisen, wie dem Schwabenkrieg, diese zunehmende Bindung an den König ein Politikum. ${ }^{183}$ Aus diesem Grund nutzte Maximilian vermutlich das beschriebene Mandat, um die Formierung der angedachten Verhaltensprämissen und die Verbreitung von bestimmten Sinnhorizonten voranzutreiben. ${ }^{184}$ Diese Handlungen könnten auch in Verbindung zur von Metz beschriebenen „informellen Politik“ der Habsburger in den Vorlanden stehen. ${ }^{185}$

Um den Kreis der Analyse zu schließen, muss in einem letzten Punkt auf die Wirkung des Mandates Rücksicht genommen werden. Dieser Punkt ist wahrscheinlich der mit Abstand am Schwersten zu bestimmende. Die Problematik resultiert dabei aus der defizitären Überlieferungslage, womit auf eine direkte Wirkung des Mandates geschlossen werden könnte. Zwar sind einzelne Reaktionen wie die der Stadt Nürnberg tradiert, es wäre jedoch zu kurz gegriffen, anhand solcher Überlieferungen Zusammenhänge herzustellen. Dennoch darf die Wirkung des Mandates nicht unterschätzt werden, so vermerkt Ulrich Strauss in einem Brief an die Stadt Nördlingen: „Seit dem letzten Mandat ziehen einige Städte mit Volk stärker zu."186 Selbiges vermerkt auch Wernher Sachs: „Dies sei nicht allein auf Bitte des Bundes, sondern wegen der schweren königlichen Mandate [geschehen], [...]."187 Somit könnte das Mandat Maximilians durchaus bis zu einem gewissen Teil seine Wirkung erzielt haben. So wandte der Bund noch vor der Aussendung kaum seine gesamte militärische Macht auf. Ab Mai 1499 lassen sich jedoch zusehends stärkere süddeutsche Kontingente feststellen. ${ }^{188}$

181 Bayerische Akademie der Wissenschaften. Historische Kommission (Hrsg.), Die Chroniken der fränkischen Städte. Nürnberg, Bd. 5 (Die Chroniken der deutschen Städte vom 14. bis ins 16. Jahrhundert 11), Leipzig 1874, S. 606-607; Ebenfalls für Memmingen: Christoph Schorer, Memminger Chronick, oder kurtze Erzehlung vieler denckwuerdigen Sachen, die sich allda nicht allein vor alten, sondern auch zu jetzigen Zeiten, bevorab in verwichenem dreyssigjaehrigen Krieg begeben und zugetragen: von Ao. 369 biß 1660. sampt einer kurtzen Beschreibung der Statt neben jhrem Grundriß in Kupffer, Ulm 1660, S. 57.

182 Adolf Layer/Wolfgang Wüst, Die habsburgischen Besitzungen. Schwäbisch-Österreich und die Vorlande, in: Max Spindler/Andreas Kraus (Hrsg.), Geschichte Schwabens bis zum Ausgang des 18. Jahrhunderts (Handbuch der bayerischen Geschichte III/2), S. 347-363, hier S. 347-348.

183 Franz Quarthal, „Die besten, getreuesten und anhänglichsten Untertanen“. Zur Geschichte der schwäbischösterreichischen Landstände, in: Beiträge zur Landeskunde (1979), Heft 1, S. 1-13, hier S. 4.

184 Zur kontextunabhängigen Verbreitung von Sinn: Schlögl, Politik, S. 587.

185 Metz, Stände, S. 35.

186 Ulrich Strauss zu Ueberlingen an Nördlingen. 30. Mai 1499, in: Klüpfel, Urkunden zur Geschichte des Schwäbischen Bundes (1488-1533). Erster Theil, 1488-1506 (Bibliothek des Literarischen Vereins in Stuttgart 14), Stuttgart 1846, S. 340-341, hier S. 341.

187 Wernher Sachs an Esslingen. 1. Juni 1499, in: Klüpfel, Urkunden zur Geschichte des Schwäbischen Bundes (1488-1533). Erster Theil, 1488-1506 (Bibliothek des Literarischen Vereins in Stuttgart 14), Stuttgart 1846, S. 341342, hier S. 342.

188 Carl, Schwabenkrieg, S. 121. 


\section{Fazit}

„Seine Schreiben sind ausführlich, angenehm, lebhaft; einseitig, aber auf ihre Weise wahr; sie bilden das sonderbarste Compendium der europäischen Geschichte jener Zeit, von dem Standpunct eines vielthätigen, hochstrebenden Fürsten aus, der jedermann in sein Interesse zu ziehen sucht."189

Auf diese Weise beschreibt Leopold Ranke die von Maximilian ausgesandten Schreiben. Auch Diederichs hat die Bewertung von Ranke als Ausgangspunkt für die Beschreibung der maximilianischen Publizistik hergenommen. ${ }^{190}$ Diese Einschätzung gewinnt hinsichtlich des Schwabenkrieges neue Relevanz, weshalb im Zuge dieser Arbeit die Frage nach der propagandistischen Dimension der publizierten Mandate gefragt wurde. Eingangs wurde deshalb zunächst vermutet, dass Maximilian durch die publizierte Propaganda seine Autorität ausnützend Einfluss auf die adressierten Stände gewinnen wollte, um sie zu einer verstärkten Teilnahme an einem Konflikt zu bewegen, der sich hauptsächlich auf das habsburgische Territorium beschränkte. Von zentraler Bedeutung waren dabei folgende Argumente: Gemäß der genuinen Kommunikationssituation der Frühen Neuzeit wäre es verfehlt moderne Mechanismen der Kommunikation direkt in die Vergangenheit zu projizieren. Vielmehr müssen die jeweiligen Rahmenbedingungen an den gewählten Zeitausschnitt angepasst werden. Daraus ergeben sich bestimmte methodische Probleme, die vor allem auf die schwierige Überlieferungslage zurückzuführen sind.

In einer ähnlichen Tradition präsentiert sich das behandelte Mandat. Zwar ist es möglich, die Quelle im Wesentlichen als Propaganda zu definieren, jedoch sind einige Anführungen bezüglich der Überlieferung, des Adressatenkreises und der Autorenschaft kritisch zu hinterfragen. Nichtsdestotrotz konnte gemessen an den primär formulierten Forschungsfragen konstatiert werden, dass Maximilian innerhalb der Kommunikation die Formierung handlungsrelevanter Meinungen und Einstellungen durch die Herstellung von Öffentlichkeit erreicht hat. Zuletzt muss betont werden, dass diese königliche Handlung, vor allem vor dem Hintergrund einer landesherrschaftlichen Durchdringung der Vorlande, betrachtet werden muss. Hinzu kommt die Tatsache, dass durch die Manipulation der Darstellung, die einen stark landesherrschaftlichen Aspekt aufweist, eine Sinn-Aktualisierung für die an der Kommunikation beteiligten Akteur*innen versucht wurde. Schlussendlich könnte daraus geschlossen werden, dass die politische Kommunikation zugunsten der Stellung Habsburgs in den Vorlanden betrieben wurde. Das Resultat dieser Bemühungen war die Reaktion auf das Mandat Maximilians vonseiten der adressierten Akteur*innen, die zum Teil konkrete Handlungen ergriffen oder auf das Mandat reagierten. Anscheinend war sich Maximilian der tragenden Rolle der Kommunikation für seine politischen Ambitionen im Schwabenkrieg bewusst. 


\section{Abkürzungen}

DRW Deutsches Rechtswörterbuch

EdN Enzyklopädie der Neuzeit

\section{Literatur}

Auerbach, Jonathan/Castronovo, Russ, Introduction: Thirteen Propositions about Propaganda, in: Jonathan Auerbach/Russ Castronovo (Hrsg.), The Oxford handbook of propaganda studies, New York 2013, S. 1-18.

Auer, Leopold, Reichshofkanzlei, in: EdN, Bd. 10, Stuttgart 2009, S. 911-914.

Baraldi, Claudio/Corsi, Giancarlo/Esposito, Elena (Hrsg.), GLU. Glossar zu Niklas Luhmanns Theorie sozialer Systeme, Frankfurt am Main $2015^{8}$.

Beck, Klaus, Kommunikation, in: Günter Bentele/Hans-Bernd Brosius/Otfried Jarren (Hrsg.), Lexikon Kommunikations- und Medienwissenschaft (Studienbücher zur Kommunikations- und Medienwissenschaft), Wiesbaden 201322, S. 155-156.

Ders., Kommunikationsforschung, in: Bentele/Brosius/Jarren (Hrsg.), Lexikon Kommunikations- und Medienwissenschaft (Studienbücher zur Kommunikations- und Medienwissenschaft), Wiesbaden 20132, S. 158-159.

Behringer, Wolfgang, Im Zeichen des Merkur. Reichspost und Kommunikationsrevolution in der Frühen Neuzeit (Veröffentlichungen des Max-Planck-Instituts für Geschichte 189), Göttingen 2003.

Ders., Kommunikation, in: EdN, Bd. 6, Stuttgart 2007, Sp. 995-1018.

Bock, Ernst, Der Schwäbische Bund und seine Verfassungen. 1488-1534, Ein Beitrag zur Geschichte der Zeit der Reichsreform (Untersuchungen zur deutschen Staats- und Rechtsgeschichte A.F. 137), Aalen $1968^{2}$.

Braun, Bettina, Die Habsburger und die Eidgenossen im späten Mittelalter, in: Irmgard Christa Becker (Hrsg.), Vorderösterreich, nur die Schwanzfeder des Kaiseradlers? Die Habsburger im deutschen Südwesten, Ulm 1999, S. 129-145.

Brückner, Wolfgang, Einblattdruck, in: EdN, Bd. 3, Stuttgart 2006, Sp. 118-120.

Ders., Flugschrift, in: EdN, Bd. 3, Stuttgart 2006, Sp. 1027-1032.

Burkhardt, Johannes/Werkstetter, Christine, Die Frühe Neuzeit als Medienzeitalter und ihr kommunikatives Spektrum. Einleitung, in: Johannes Burkhardt/Christine Werkstetter (Hrsg.), Kommunikation und Medien in der Frühen Neuzeit (HZ. Beihefte n.F. 41), München 2005, S. 1-7.

Burkhardt, Johannes/Werkstetter, Christine (Hrsg.), Begann die Neuzeit mit dem Buchdruck? Ist die Ära der Typographie im Zeitalter der digitalen Medien endgültig vorbei? Podiumsdiskussion unter der Leitung von Winfried Schulze, in: Burkhardt/Werkstetter 
(Hrsg.), Kommunikation und Medien in der Frühen Neuzeit (HZ. Beihefte n.F. 41), München 2005, S. 11-39.

Bussemer, Thymian, Propaganda. Konzepte und Theorien, Wiesbaden 2005.

Carl, Horst, "Schwabenkrieg“ oder "Schweizerkrieg"? Der Schwäbische Bund als Gegner der Eidgenossenschaft, in: Historischer Verein des Kantons Solothurn (Hrsg.), Gedenkschrift 500 Jahre Schlacht bei Dornach 1499-1999 (Jahrbuch für solothurnische Geschichte 72), Solothurn 1999, S. 97-130.

Ders., Der Schwäbische Bund 1488-1534. Landfrieden und Genossenschaft im Übergang vom Spätmittelalter zur Reformation (Schriften zur südwestdeutschen Landeskunde 24), Leinfelden 2000.

Ders., Landfrieden als Konzept und Realität kollektiver Sicherheit im Heiligen Römischen Reich, in: Gisela Naegle (Hrsg.), Frieden schaffen und sich verteidigen im Spätmittelalter. Faire la paix et se défendre à la fin du Moyen Âge (Pariser Historische Studien 98), München 2012, S. 121-138.

Crivellari, Fabio/Kirchmann, Kay u. a. Einleitung: Die Medialität der Geschichte und die Historizität der Medien, in: Fabio Crivellari/Sven Grampp u. a., (Hrsg.), Die Medien der Geschichte. Historizität und Medialität in interdisziplinärer Perspektive (Historische Kulturwissenschaft 4), Konstanz 2004, S. 9-48.

Diederichs, Peter, Kaiser Maximilian I. als politischer Publizist, Heidelberg 1933.

Doering-Manteuffel, Sabine, Informationsstrategien: Propaganda, Geheimhaltung, Nachrichtennetze. Einleitung, in: Burkhardt/Werkstetter (Hrsg.), Kommunikation und Medien in der Frühen Neuzeit (HZ. Beihefte n.F. 41), München 2005, S. 367-384.

Ecker, Gisela, Einblattdrucke von den Anfängen bis 1555. Untersuchungen zu einer Publikationsform literarischer Texte (Göppinger Arbeiten zur Germanistik 314), Göppingen 1981.

Eisenstein, Elizabeth L., The printing revolution in early modern Europe, Cambridge $2005^{2}$.

Eisermann, Falk, Auflagenhöhe von Einblattdrucken im 15. und frühen 16. Jahrhundert, in: Volker Honemann (Hrsg.), Einblattdrucke des 15. und frühen 16. Jahrhunderts. Probleme, Perspektiven, Fallstudien, Tübingen 2000, S. 143-178.

Ders., Bevor die Blätter fliegen lernten. Buchdruck, politische Kommunikation und die ,Medienrevolution' des 15. Jahrhunderts, in: Karl-Heinz Spieß/Oliver Auge (Hrsg.), Medien der Kommunikation im Mittelalter (Beiträge zur Kommunikationsgeschichte 15), Stuttgart 2003, S. 289-320.

Faulstich, Werner, Medien zwischen Herrschaft und Revolte. Die Medienkultur der frühen Neuzeit (1400-1700) (Die Geschichte der Medien 3), Göttingen 1998.

Frevert, Ute, Politische Kommunikation und ihre Medien, in: Ute Frevert/Wolfgang Braungart (Hrsg.), Sprachen des Politischen. Medien und Medialität in der Geschichte, Göttingen 2004, S. 7-19. 
Funk, Albert, Kleine Geschichte des Föderalismus. Vom Fürstenbund zur Bundesrepublik, Paderborn $2012^{2}$.

Füssel, Marian/Weller, Thomas, Einleitung, in: Marian Füssel/Thomas Weller (Hrsg.), Ordnung und Distinktion. Praktiken sozialer Repräsentation in der ständischen Gesellschaft (Symbolische Kommunikation und gesellschaftliche Wertesysteme 8), Münster 2005, S. $9-22$.

Füssel, Stephan, Der Theuerdank von 1517. Kaiser Maximilian und die Medien seiner Zeit, Eine kulturhistorische Einführung von Stephan Füssel, München 2003.

Gawlik, Alfred, Litterae, in: Lexikon des Mittelalters, Bd. V, Stuttgart 1999, Sp. 2023-2024.

Ders., Mandat, in: Lexikon des Mittelalters, Bd. VI, Stuttgart 1999, Sp. 186-187.

Giesecke, Michael, Der Buchdruck in der frühen Neuzeit. Eine historische Fallstudie über die Durchsetzung neuer Informations- und Kommunikationstechnologien, Frankfurt am Main $2006^{4}$.

Grotefend, Hermann, Zeitrechnung des deutschen Mittelalters und der Neuzeit. Glossar und Tafeln, Bd. 1, Hannover 1891.

Gutmann, Andre, Die Schwabenkriegschronik des Kaspar Frey und ihre Stellung in der eidgenössischen Historiographie des 16. Jahrhunderts (Veröffentlichungen der Kommission für Geschichtliche Landeskunde in Baden-Württemberg Reihe B, Forschungen 176,1), Stuttgart 2010.

Habermas, Jürgen, Strukturwandel der Öffentlichkeit. Untersuchungen zu einer Kategorie der bürgerlichen Gesellschaft, Frankfurt am Main $2015^{14}$.

Hecht, Winfried, Rottweil ca. 1340-1529. Im Herbst des Mittelalters, Rottweil 2005.

Heidelberger Akademie der Wissenschaften (Hrsg.), Mandat, in: DRW, Bd. 9, Stuttgart 1996, Sp. 100-103.

Heimann, Heinz-Dieter, Die Habsburger. Dynastie und Kaiserreiche, München $2001^{5}$.

Henkel, Nikolaus, Ein unveröffentlichtes deutsches Flugblatt Sebastian Brants. Die Klage des Friedens gegen den Krieg und die Verteidigung des Kriegs gegen den Frieden, in: Rudolf Bentzinger (Hrsg.), Grundlagen: Forschungen, Editionen und Materialien zur deutschen Literatur und Sprache des Mittelalters und der Frühen Neuzeit (Zeitschrift für deutsches Altertum und deutsche Literatur, Beiheft 18), Stuttgart 2013, S. 523-534. Hollegger, Manfred, Maximilian I. Herrscher und Mensch einer Zeitenwende, Stuttgart 2005.

Honecker, Patrick, Vorreformatorische Schlagwörter. Spiegel politischer, religiöser und sozialer Konflikte in der frühen Neuzeit, phil. Diss. Trier 2002.

Honemann, Volker, Vorformen des Einblattdruckes. Urkunden - Schrifttafeln - Textierte Tafelbilder - Anschläge - Einblatthandschriften, in: Honemann (Hrsg.), Einblattdrucke 
des 15. und frühen 16. Jahrhunderts. Probleme, Perspektiven, Fallstudien, Tübingen 2000, S. 1-44.

Jaeger, Friedrich, Kultur, in: EdN, Bd. 7, Stuttgart 2008, Sp. 253-281.

Kerth, Sonja, Der landsfrid ist zerbrochen. Das Bild des Krieges in den politischen Ereignisdichtungen des 13. bis 16. Jahrhunderts (Imagines medii aevi 1), Wiesbaden 1997.

Krieb, Steffen, Vom Totengedenken zum politischen Argument. Die Schlacht bei Sempach (1386) im Gedächtnis des Hauses Habsburg und des südwestdeutschen Adels im 15. Jahrhundert, in: Dieter Langewiesche/Friedrich Lenger u. a. (Hrsg.), Kriegsniederlagen. Erfahrung und Erinnerung, Berlin 2015, S. 69-88.

Lasswell, Harold D., The structure and function of communication in society, in: Lyman Bryson (Hrsg.), The Communications of Ideas, New York 1948, S. 117-130.

Layer, Adolf/Wüst, Wolfgang, Die habsburgischen Besitzungen. Schwäbisch-Österreich und die Vorlande, in: Max Spindler/Andreas Kraus (Hrsg.), Geschichte Schwabens bis zum Ausgang des 18. Jahrhunderts (Handbuch der bayerischen Geschichte III/2), S. 347-363.

Luhmann, Niklas, Soziale Systeme. Grundriss einer allgemeinen Theorie, Frankfurt am Main $1987^{2}$.

MacLuhan, Herbert Marshall/Fiore, Quentin, The medium is the massage. Inventory of effects, Harmonsworth 1967.

Merten, Klaus, Struktur und Funktion von Propaganda, in: Publizistik 45 (2000), Heft 2, S. 143-162.

Metz, Axel, Der Stände oberster Herr. Königtum und Landstände im süddeutschen Raum zur Zeit Maximilians I. (Veröffentlichungen der Kommission für Geschichtliche Landeskunde in Baden-Württemberg Reihe B, Forschungen 174), Stuttgart 2009.

Meyer, Werner, Krisen, Korruption und Kampfbegierde. Der politische, ideologische und emotionale Konfliktrahmen des Schwabenkrieges von 1499, in: Historischer Verein des Kantons Solothurn (Hrsg.), Gedenkschrift 500 Jahre Schlacht bei Dornach 1499-1999 (Jahrbuch für solothurnische Geschichte 72), Solothurn 1999, S. 10-52.

Mommsen, Karl, Eidgenossen, Kaiser und Reich. Studien zur Stellung der Eidgenossenschaft innerhalb des Heiligen Römischen Reiches (Basler Beiträge zur Geschichtswissenschaft 72), Stuttgart-Basel 1958.

Müller, Jan-Dirk, Publizistik unter Maximilian I. Zwischen Buchdruck und mündlicher Verkündigung, in: Ute Frevert/Wolfgang Braungart (Hrsg.), Sprachen des Politischen. Medien und Medialität in der Geschichte, Göttingen 2004, S. 95-122.

Ders., Einleitung, in: Jan-Dirk Müller/Hans-Joachim Ziegeler (Hrsg.), Maximilians Ruhmeswerk. Künste und Wissenschaften im Umkreis Kaiser Maximilians I., Berlin 2015, S. 1-6.

Niederhäuser, Peter/Sennhauser, Raphael, Kaiser Maximilian I. und die Eidgenossen. Kunst und Propaganda des «letzten Ritters», in: Peter Niederhäuser (Hrsg.), Vom „Frei- 
heitskrieg" zum Geschichtsmythos. 500 Jahre Schweizer- oder Schwabenkrieg, Zürich 2000, S. 73-102.

Niederstätter, Alois, Österreich wider den «Erbfeind aller Ritterschaft, allen Adels und aller Ehrbarkeit». Zur Rolle Maximilians I. und seiner Räte im Schwabenkrieg, in: Historischer Verein des Kantons Solothurn (Hrsg.), Gedenkschrift 500 Jahre Schlacht bei Dornach 1499-1999 (Jahrbuch für solothurnische Geschichte 72), Solothurn 1999, S. $133-173$.

Ders., Geschichte Vorarlbergs. Vorarlberg im Mittelalter, Bd. 1, Innsbruck 2014.

Press, Volker, Die Erblande und das Reich von Albrecht II. bis Karl VI. (1438-1740), in: Robert A. Kann/Friedrich E. Prinz (Hrsg.), Deutschland und Österreich. Ein bilaterales Geschichtsbuch, Wien-München 1980, S. 44-88.

Ders., Vorderösterreich in der habsburgischen Reichspolitik des späten Mittelalters und der frühen Neuzeit, in: Hans Maier (Hrsg.), Vorderösterreich in der frühen Neuzeit, Sigmaringen 1989, S. 1-42.

Quarthal, Franz, „Die besten, getreuesten und anhänglichsten Untertanen“. Zur Geschichte der schwäbisch-österreichischen Landstände, in: Beiträge zur Landeskunde (1979), Heft 1, S. 1-13.

Ders., Die habsburgischen Landstände in Südwestdeutschland, in: Günther Bradler (Hrsg.), Von der Ständeversammlung zum demokratischen Parlament. Die Geschichte der Volksvertretungen in Baden-Württemberg, Stuttgart 1987, S. 79-92.

Ranke, Leopold, Deutsche Geschichte im Zeitalter der Reformation, Bd. 6, Berlin 1847.

Reinfandt, Christoph, Systemtheorie, in: Ansgar Nünning (Hrsg.), Metzler Lexikon Literatur- und Kulturtheorie. Ansätze - Personen - Grundbegriffe, Stuttgart-Weimar 2013, S. $735-737$.

Reinhard, Wolfgang, Rezension zu: Johannes Burkhardt/Christine Werkstetter (Hrsg.), Kommunikation und Medien in der Frühen Neuzeit (HZ. Beihefte. NF, 41), München 2005, in: Zeitschrift für Historische Forschung 34 (2007), Heft 4, S. 684-686.

Reinhardt, Volker, Die Geschichte der Schweiz. Von den Anfängen bis heute, München 2014.

Rogg, Matthias, Landsknecht, in: EdN, Bd. 7, Stuttgart 2008, Sp. 559-561.

Schaab, Meinrad, Spätmittelalter (1250-1500), in: Meinrad Schaab/Hansmartin Schwarzmaier (Hrsg.), Allgemeine Geschichte. Teil 2, Vom Spätmittelalter bis zum Ende des Alten Reiches (Handbuch der baden-württembergischen Geschichte 1), Stuttgart 2000, S. 1-144.

Schadek, Hans, Der Kaiser und seine Stadt. Maximilian I. und seine Beziehung zu Freiburg, in: Hans Schadek (Hrsg.), Der Kaiser in seiner Stadt. Maximilian I. und der Reichstag zu Freiburg 1498 (Zeitschrift des Breisgau-Geschichtsvereins Schau-ins-Land 117), Freiburg im Breisgau 1998, S. 216-273. 
Schieder, Wolfgang/Dipper, Christof, Propaganda, in: Otto Brunner/Werner Conze/ Reinhart Koselleck (Hrsg.), Geschichtliche Grundbegriffe. Historisches Lexikon zur politisch-sozialen Sprache in Deutschland, Bd. 5, Stuttgart 1984, S. 69-88.

Schilling, Michael, Bildpublizistik der frühen Neuzeit. Aufgaben und Leistungen des illustrierten Flugblatts in Deutschland bis um 1700 (Studien und Texte zur Sozialgeschichte der Literatur 29), Tübingen 1990.

Schlögl, Rudolf, Kommunikation und Vergesellschaftung unter Anwesenden. Formen des Sozialen und ihre Transformation in der Frühen Neuzeit, in: Geschichte und Gesellschaft 34 (2008), Heft 2, S. 155-224.

Ders., Medien der Macht und des Entscheidens. Schrift und Druck im politischen Raum der europäischen Vormoderne (14.-17. Jahrhundert), Eine Einleitung, in: Jan Marco Sawilla/Rudolf Schlögl (Hrsg.), Medien der Macht und des Entscheidens. Schrift und Druck im politischen Raum der europäischen Vormoderne (14.-17. Jahrhundert) (The formation of Europe 5), Hannover 2014, S. 7-32.

Ders., Politik beobachten. Öffentlichkeit und Medien in der Frühen Neuzeit, in: Zeitschrift für Historische Forschung 35 (2008), Heft 4, S. 581-616.

Ders., Anwesende und Abwesende. Grundriss für eine Gesellschaftsgeschichte der Frühen Neuzeit, Konstanz 2014.

Schmale, Wolfgang/Zimmermann, Clemens/Mahlerwein, Gunter, Öffentlichkeit, in: EdN, Bd. 9, Stuttgart 2009, Sp. 358-367.

Schnurr, Eva-Maria, Religionskonflikt und Öffentlichkeit. Eine Mediengeschichte des Kölner Kriegs (1582 bis 1590) (Rheinisches Archiv 154), Köln 2009.

Schuler, Peter-Johannes, Schwäbischer Bund, in: Lexikon des Mittelalters, Bd. VII, Sp. 1607-1608.

Schulze, Winfried, Politische Kommunikation. Theoretische Ansätze und Ergebnisse empirischer Forschung, Wiesbaden $2011^{3}$.

Sieber-Lehmann, Claudius/Bertin, Christian/Wilhelmi, Thomas (Hrsg.), In Helvetios-wider die Kuhschweizer. Fremd- und Feindbilder von den Schweizern in antieidgenössischen Texten aus der Zeit von 1386 bis 1532 (Schweizer Texte n.F. 13), Bern 1998.

Sieber-Lehmann, Claudius, Einleitung, in: Sieber-Lehmann/Bertin/Wilhelmi (Hrsg.), In Helvetios-wider die Kuhschweizer., S. 1-22.

Speck, Dieter, Kleine Geschichte Vorderösterreichs, Karlsruhe $2016^{2}$.

Stauber, Reinhard, Maximilian I. als ,politischer Virtuose', in: Jan-Dirk Müller/Hans-Joachim Ziegeler (Hrsg.), Maximilians Ruhmeswerk. Künste und Wissenschaften im Umkreis Kaiser Maximilians I., Berlin 2015, S. 7-30.

Stettler, Bernhard, Sempach, Schlacht bei, in: Lexikon des Mittelalters, Bd. VII, Sp. 1742. 
Stollberg-Rilinger, Barbara, Das Heilige Römische Reich Deutscher Nation, München $2013^{5}$.

Dies., Des Kaisers alte Kleider. Verfassungsgeschichte und Symbolsprache des Alten Reiches, München $2013^{2}$.

Studt, Birgit, Geplante Öffentlichkeit. Propaganda, in: Konstanzer Arbeitskreis für mittelalterliche Geschichte (Hrsg.), politische Öffentlichkeit im Spätmittelalter (Vorträge und Forschungen 75), Konstanz 2011, S. 203-236.

Vowe, Gerhard, Politische Kommunikation, in: Bentele/Hans-Bernd Brosius/Otfried Jarren (Hrsg.), Lexikon Kommunikations- und Medienwissenschaft, Wiesbaden 20132, S. 266-268.

Wiesflecker, Hermann/Beer, Christa u. a. (Hrsg.), Regesta Imperii, 1499-1501, Bd. XIV/3 Teil 1: Maximilian I. 1499-1501, Wien-Köln-Weimar 1996.

Wiesflecker, Hermann, Österreich im Zeitalter Maximilians I. Die Vereinigung der Länder zum frühmodernen Staat, der Aufstieg zur Weltmacht, Wien 1999.

Würgler, Andreas, Medien in der frühen Neuzeit (Enzyklopädie deutscher Geschichte 85), München 2009.

Wüst, Wolfgang, Die Adelskurien. Zwischen vorderösterreichischer Landsässigkeit, ständischer Autonomie und Reichsfreiheit: Die »|nsassen« in der Markgrafschaft Burgau, die »Anstösser« in der Landvogtei Schwaben und die Hegauer Reichsritter in der Landgrafschaft Nellenburg, Ein Vergleich, in: Becker (Hrsg.), Vorderösterreich, nur die Schwanzfeder des Kaiseradlers? Die Habsburger im deutschen Südwesten, Ulm 1999, S. 188-195.

Wuttke, Dieter, Ein unbekannter Brief von Willibald Pirckheimer, in: Archiv für Kulturgeschichte 50 (1968), S. 294-299.

Zimmermann, Clemens, Medien, in: EdN, Bd. 8, Stuttgart 2008, Sp. 223-243.

\section{Quellen}

Anonymus, Spottlied der Landsknechte gegen die Eidgenossen. Januar 1499, in: Sieber-Lehmann/Bertin/Wilhelmi (Hrsg.), In Helvetios-wider die Kuhschweizer. Fremdund Feindbilder von den Schweizern in antieidgenössischen Texten aus der Zeit von 1386 bis 1532 (Schweizer Texte n.F. 13), Bern 1998, S. 85-87.

Anshelm, Valerius, Die Berner-Chronik, Bd. 2, herausgegeben von Historischen Verein des Kantons Bern, Bern 1886

Aristoteles, Rhetorik, übersetzt und herausgegeben von Gernot Krapinger, Stuttgart $2007^{2}$.

Bayerische Akademie der Wissenschaften, Historische Kommission (Hrsg.), Die Chroniken der fränkischen Städte. Nürnberg, Bd. 5 (Die Chroniken der deutschen Städte vom 14. bis ins 16. Jahrhundert 11), Leipzig 1874. 
M2205005, Maximilian I., Römischer Kaiser: Mahnung an die Reichsstände, Hilfe gegen die Eidgenossen und die Graubündner zu leisten, Formular für Fürsten, Freiburg im Breisgau 22. IV. 1499. Staatsbibliothek zu Berlin, Stiftung Preußischer Kulturbesitz, [https://www.gesamtkatalogderwiegendrucke.de/], eingesehen 24. 3. 2019.

M22051, Maximilian I., Römischer Kaiser: Mahnung an die Reichsstände, Hilfe gegen die Eidgenossen und die Graubündner zu leisten. Freiburg im Breisgau 22. IV. 1499. Staatsbibliothek zu Berlin, Stiftung Preußischer Kulturbesitz, [https://www.gesamtkatalogderwiegendrucke.de/pics/22051.jpg], eingesehen 24. 3. 2019.

M2205110, Maximilian I., Römischer Kaiser: Mahnung an die Reichsstände, Hilfe gegen die Eidgenossen und die Graubündner zu leisten, Formular für adlige Stände, Freiburg im Breisgau 22. IV. 1499. Staatsbibliothek zu Berlin, Stiftung Preußischer Kulturbesitz, [https://www.gesamtkatalogderwiegendrucke.de/pics/2205110.jpg], eingesehen 24. 3. 2019.

M22052, Maximilian I., Römischer Kaiser: Mahnung an die Reichsstände, Hilfe gegen die Eidgenossen und die Graubündner zu leisten, Formular für besondere Fälle, Freiburg im Breisgau 22. IV. 1499. Staatsbibliothek zu Berlin, Stiftung Preußischer Kulturbesitz, [https://www.gesamtkatalogderwiegendrucke.de/pics/22052.jpg], eingesehen 24. 3. 2019.

Mappe Einbl. (Ohly-Sack 1936) - Mahnung an die Reichsstände, Hilfe gegen die Eidgenossen und die Graubündner zu leisten, Freiburg im Breisgau 22. IV. 1499. Universitätsbibliothek Frankfurt am Main, [http://sammlungen.ub.uni-frankfurt.de/inc/urn/ urn:nbn:de:hebis:30:2-241789], eingesehen 24. 3. 2019.

Maximilian I. Römischer König, Mahnung an die Reichsstände, Hilfe gegen die Eidgenossen und die Graubündner zu leisten, Freiburg, 22. IV. 1499 [Mainz, Peter Schöffer, vor 5. V. 1499], Mappe Einblattdrucke 981, Stadt- und Universitätsbibliothek Frankfurt am Main, auffindbar unter Nr. 1936 in: Kurt Ohly/Vera Sack (Hrsg.), Inkunabelkatalog der Stadt- und Universitätsbibliothek und anderer öffentlicher Sammlungen in Frankfurt am Main, Bd. 1, Frankfurt am Main 1967, S. 357.

Maximilian I. Römischer König, Mandat dasz dem pund zugetzogen werden soll. 23. April 1499, in: Klüpfel, Urkunden zur Geschichte des Schwäbischen Bundes (1488-1533). Erster Theil, 1488-1506 (Bibliothek des Literarischen Vereins in Stuttgart 14), Stuttgart 1846, S. 321-322.

Reichs-Regiment, in: Johann Heinrich Zedler (Hrsg.), Grosses vollständiges Universal Lexicon Aller Wissenschafften und Künste, Bd. 31, Halle-Leipzig 1742, Sp. 163-165.

Schorer, Christoph, Memminger Chronick, oder kurtze Erzehlung vieler denckwuerdigen Sachen, die sich allda nicht allein vor alten, sondern auch zu jetzigen Zeiten, bevorab in verwichenem dreyssigjaehrigen Krieg begeben und zugetragen: von Ao. 369 biß 1660. sampt einer kurtzen Beschreibung der Statt neben jhrem Grundriß in Kupffer, UIm 1660. 
Ulrich Strauss zu Ueberlingen an Nördlingen. 30. Mai 1499, in: Klüpfel, Urkunden zur Geschichte des Schwäbischen Bundes (1488-1533). Erster Theil, 1488-1506 (Bibliothek des Literarischen Vereins in Stuttgart 14), Stuttgart 1846, S. 340-341.

Urkunden zur Geschichte des Schwäbischen Bundes (1488-1533), herausgegeben von Karl Klüpfel, Stuttgart 1846.

Wernher Sachs an Esslingen. 1. Juni 1499, in: Klüpfel, Urkunden zur Geschichte des Schwäbischen Bundes (1488-1533). Erster Theil, 1488-1506 (Bibliothek des Literarischen Vereins in Stuttgart 14), Stuttgart 1846, S. 341-342.

Tobias Riedmann ist Student der Geschichtswissenschaft an der Universität Innsbruck. tobias.riedmann@student.uibk.ac.at

\section{Zitation dieses Beitrages}

Tobias Riedmannner "den fliegenden maren zeglauben kein ursach sîe" - Das Mandat Maximilians I. vom 22. April 1499 in seiner propagandistischen Dimension, in: historia.scribere 11 (2019), S. 177-212, [http://historia.scribere.at], eingesehen 17.6.2019 (=aktuelles Datum). 
